\title{
Energy Efficient Uplink Transmission in Cooperative mmWave NOMA Networks with Wireless Power Transfer
}

\author{
Azadeh Khazali, Daniele Tarchi, Senior Member, IEEE, Mahrokh G. Shayesteh, Senior Member, IEEE, \\ Hashem Kalbkhani Member, IEEE, and Arash Bozorgchenani, Member, IEEE
}

\begin{abstract}
In 5G wireless networks, cooperative non-orthogonal multiple access (NOMA) and wireless power transfer (WPT) are efficient ways to improve the spectral efficiency (SE) and energy efficiency (EE). In this paper, a new cooperative NOMA scheme with WPT is proposed, where EE optimization with a constrained maximum transmit power and minimum required $\mathrm{SE}$ is considered for the user grouping and transmit power allocation of users. We obtain a sub-optimal solution by decoupling the original problem in two sub-problems: an iterative algorithm is considered for the user grouping, while, in addition, we utilize the Bat Algorithm (BA) for solving the power allocation problem, where BA was proved to be able to achieve a higher accuracy and efficiency with respect to other meta-heuristic algorithms. Furthermore, to validate the performance of the proposed system, analytical expressions for the energy outage probability and outage probability of users are derived, confirming the effectiveness of the simulation results. It is demonstrated that the proposed cooperative NOMA with WPT offers a considerable improvement in terms of $\mathrm{SE}$ and $\mathrm{EE}$ of the network compared to other methods. Finally, the effectiveness of $\mathrm{BA}$ in solving the EE optimization problem is demonstrated through a high convergence speed by comparing it with other methods.
\end{abstract}

Index Terms-- Bat Algorithm, Cooperative NOMA, Energy efficiency, mmWave network, Spectral efficiency, Wireless power transfer.

\section{INTRODUCTION}

$\mathrm{T}$ HE demand for high data rate has increased rapidly in the last decade. This rapid growth in mobile data traffic should be accompanied with the same growth in the energy efficiency (EE) and spectral efficiency (SE) of the network [1]. To this end, several technologies have been introduced. One of the

A. Khazali and M. G. Shayesteh are with the Department of Electrical and Computer Engineering, Urmia University, Urmia, Iran. M. G. Shayesteh is also working with the Wireless Research Lab., ACRI, Department of Electrical Engineering, Sharif University of Technology, Tehran, Iran (e-mail: a.khazali@urmia.ac.ir, m.shayesteh@urmia.ac.ir).

D. Tarchi is with the Department of Electrical, Electronic and Information Engineering, University of Bologna, Italy (e-mail: daniele.tarchi@unibo.it).

H. Kalbkhani is with the Faculty of Electrical Engineering, Urmia University of Technology, Urmia, Iran (e-mail: h.kalbkhani@uut.ac.ir).

A. Bozorgchenani was with the Department of Electrical, Electronic and Information Engineering, University of Bologna, Italy. He is now with the Department of Computing and Communications, Lancaster University, UK (e-mail: a.bozorgchenani@Lancaster.ac.uk)

A major part of the work was done during A. Khazali's visit to the University of Bologna, Italy. most promising technologies is millimeter-Wave (mmWave) communication which provides wide bandwidth and high SE. However, the deployment of mmWave in long-range communications is challenging due to the high path loss, low penetration, and high sensitivity to blockage. As stated in [2], SE and EE improvement of the network can also be afforded by resorting to the deployment of small-cell networks (SCNs). Short-range SCNs complement the macro-cell networks to provide coverage for both indoor and outdoor wireless networks [3, 4]. SCN is also an effective technology for deploying mmWave, therefore, a promising approach in $5 \mathrm{G}$ for supporting high data rate demand is combining SCNs and mmWave [5]. Non-orthogonal multiple access (NOMA) has been introduced as well for enhancing SE in 5G [6,7]. The key concept in power domain NOMA is allocating each orthogonal resource block with different power levels to more than one user [6], where at the receiver side of NOMA systems, successive interference cancelation (SIC) is applied to detect the desired signal $[8,9]$. Moreover, to improve the transmission reliability of users with poor channel gain, cooperative NOMA was introduced. Cooperative NOMA enhances the SE of the network by increasing the diversity gain [10]. Furthermore, the limited power of the mobile devices has attracted lots of attention when focusing on their impact in the cooperative wireless networks.

Beside allocating the optimum power, when minimizing the power consumption or maximizing the EE of the system, another method for increasing the lifetime of network is energy harvesting from radio frequency (RF) [11,12]. Wireless power transfer (WPT) is an RF energy harvesting technology attracting the interests of researchers nowadays. Applying WPT in cooperative NOMA is an efficient method for increasing the coverage and lifetime of the network [13-15]. Moreover, WPT can exploit numerous advantages of the dense deployment of mmWave SCNs [16], e.g., significant reduction of the path-loss over the transmission links, improvement of frequency reuse across a region and reduction in the number of competitors for given radio resources at each SCN. As an example, in [17], the authors proved that mmWave SCNs are a viable solution for improving the WPT performance. In [18, 19], it was proved that mmWave WPT has the potential to provide better energy coverage than lower frequencies WPT. Thus, based on the aforementioned factors, the feasibility of the mmWave 
communication for the WPT application in the future $5 \mathrm{G}$ networks is ensured.

Noting the above, the coexistence of mmWave SCNs and wireless-powered communications with cooperative NOMA is a promising approach mainly due to the following advantages:

- The propagation characteristics of mmWaves made their use suitable in short range communications such as SCNs.

- Highly directional antennas in mmWave networks lead to a high correlation among users' channels which is desired in NOMA [20].

- Cooperative communication improves the SE and EE of mmWave-NOMA network.

- Integration of WPT with mmWave SCN and NOMA can further increase the EE of the network.

The aforementioned advantages have motivated us to consider the use of cooperative NOMA jointly with WPT in a mmWave SCN scenario. In addition, in order to have successful SIC at the base station (BS) for NOMA uplink communications, efficient power allocation is essential. To this end, this work aims to investigate the implementation of cooperative NOMA with WPT in the uplink mmWave SCN, for which a power allocation solution is proposed.

\section{A. Related works}

\section{1) Studies on mmWave-NOMA networks}

There are several studies that indicate the efficiency of NOMA in mmWave networks. In [21-24], the authors investigated the performance of NOMA in mmWave networks and showed the superiority of NOMA over OMA in terms of throughput. In [22], to reduce the feedback of channel state information (CSI), two random beamforming solutions were investigated. In [25], closed-form expressions of outage probability were derived for different pairing methods for machine-to-machine (M2M) communication systems in mmWave-NOMA scenarios. In [26], the benefit of coexistence of NOMA and SCN in 5G was explored. Data rate maximization problem for joint power control and beamforming of a two-user uplink mmWave-NOMA system was studied in [27]. Also in [28], an EE maximization problem for a downlink scenario with hybrid beamforming in a mmWave multiple input multiple output (MIMO) system was investigated. In [29], the superiority of NOMA over OMA in a heterogeneous network with small cell base station (SBS) was shown. The authors in [30] considered the EE maximization problem in an uplink mmWave-NOMA massive MIMO system. In [31], the sum data rate maximization problem was formulated to jointly optimize the power allocation and hybrid beamforming in a downlink mmWave-NOMA network. In [32], the authors investigated the effect of different distancebased user grouping methods on the mmWave-NOMA network performance.

\section{2) Studies on cooperative NOMA with WPT}

One of the key objectives of future $5 \mathrm{G}$ networks is maximizing the EE. Recent works on wireless power transfer show its effectiveness in improving the EE of $5 \mathrm{G}$ network. Moreover, the RF signals are able to carry both information and energy, therefore combining WPT and wireless information transmission is possible. Motivated by this, recently, the advantage of simultaneous wireless information and power transfer (SWIPT) in NOMA networks has been explored considerably. In [33], a hybrid NOMA scenario was considered; the transmission in uplink was done by exploiting the energy which is harvested in the received downlink signal. In [34], the authors investigated the effect of amplify-andforward relay in a cooperative energy harvesting NOMA scenario. In [35], an EE maximization problem of device-todevice (D2D) pairs in an energy harvesting NOMA scenario was studied. The importance of the difference between channel power gains in a NOMA with SWIPT scenario was discussed in [36]. The authors proved that when the difference is sufficiently high, NOMA outperforms OMA in a SWIPT scenario. In [37, 38], the application of SWIPT in a cooperative NOMA scenario was considered. Energy harvesting was performed by the user close to the BS acting as relay for far users. In [39], the optimization problem was applied on the data rate to obtain the optimum beamforming and power splitting ratio for the strong user, which acts as energy harvesting relay for the weak users in the cooperative NOMA scenario. In [40], the power minimization problem was studied in a SWIPTenabled NOMA scenario with time switching based receivers. In [41], the EE optimization problem was considered to jointly obtain the beamforming and power splitting ratio in a cooperative NOMA strategy.

\section{B. Motivation ad contributions}

Unlike the previous works [37-41], we consider an uplink scenario with the aim of EE maximization in a cooperative NOMA-mmWave network. To the best of our knowledge, the co-existence of cooperative NOMA with WPT, mmWave and SCN in an uplink scenario has not been considered yet. While the joint use of NOMA with mmWave in SCN has been considered, we demonstrate that by using WPT it is possible to further enhance the EE of the system. This is particularly important in case of uplink scenarios that are strongly affected by power imbalance of different users. Furthermore, while previous works were mainly based on time switching WPT, here we consider power splitting WPT. Despite power splitting requires more complex hardware implementations [42], it allows a higher SE with respect to the time switching approach, since in the latter, data and energy are orthogonally transmitted in time. Moreover, in cooperative uplink communications with power splitting methods, the source of data and energy is the device that is characterized by a limited energy battery [30, 43]. Motivated by these facts, we propose a cooperative mmWave NOMA with WPT solution for uplink transmission of small cell users (SCUs). We exploit the power splitting method such that the source of energy for both users is SBS which has no limitation in terms of energy. Moreover, in the proposed scenario, both cooperation and WPT are performed by consuming one extra time slot. The main contributions of this paper are summarized as follows:

- A new cooperative NOMA with WPT in an uplink scenario is proposed to enhance the EE and SE of the mmWave 
SCUs.

- An EE optimization method is formulated to allocate transmit powers to near and far users.

- User grouping algorithms are introduced for the proposed scenario.

- An efficient solution based on Bat Algorithm (BA) is proposed to solve the optimization problem.

- Analytical expressions are provided for energy outage probability in a non-linear energy harvesting scenario, for outage probabilities of near and far users, and diversity order of users.

The rest of this paper is organized as follows. In Section II, the system model of cooperative NOMA with SWIPT in a SCN for uplink is presented. In Section III, the proposed user grouping scheme and the power allocation method are explained. In Section IV, analytical expressions are derived for the energy outage probability and outage probabilities of the proposed system for near and far users. Numerical results are discussed in Section V and, finally, conclusions are drawn in Sections VI.

\section{SYSTEM MODEL}

We consider a cooperative mmWave-NOMA network with WPT composed of one SBS and two groups of uniformly distributed users, as $\left\{A_{i}\right\}$ and $\left\{B_{j}\right\}$. The users in group $\left\{A_{i}\right\}$ are located within the region $D_{A}$ with radius $R_{S_{A}}$ and a central angle $\Delta$ around the SBS. The users in group $\left\{B_{j}\right\}$ are located within the region $D_{B}$ with the maximum radius of $R_{S_{B}}$, minimum radius of $R_{S_{C}}$ and central angle of $\Delta$ around the SBS, where $R_{S_{A}}<R_{S_{c}}<R_{S_{B}}$.

Short distances, around a few tens of meters, between users and SBS allow the UEs to practically implement WPT. We assume that each user is equipped with an energy harvesting circuit and that the direct link between the SBS and far user is highly attenuated compared to the link between the SBS and near user, due to the higher distance. WPT technology allows to exploit a wireless signal for transferring power towards a remote UE. This is convenient in the considered scenario since the SBS is supposed to have unlimited energy while the users are battery powered. In addition, to overcome the high propagation losses of mmWave band, we assume that the SBS can transmit energy signal by exploiting $M$ transmit antennas, while, for more simplicity, we consider that each user has a single antenna. In the proposed system, we assume that the users in $\left\{A_{i}\right\}$ harvest energy from the SBS in the mmWave band and act as relay for decoding and forwarding the messages of users in $\left\{B_{j}\right\}$, where a part of the required power for relay operation is attained from the harvested energy. In addition, in the second phase, the users in $\left\{B_{j}\right\}$ harvest energy from SBS in the mmWave band. Therefore, the source of energy for both users in the proposed uplink scenario is SBS which has no limitation in energy.

The message sent by each user in $\left\{A_{i}\right\}$ is a superimposed message composed of its message and the message transmitted by its corresponding user in $\left\{B_{j}\right\}$. In the proposed model, full duplex transmission is considered and the users in $\left\{A_{i}\right\}$ exploit decode and forward relaying protocol. Perfect signal decoding is used in relay mode. In the following, we focus our analysis on two users, $U E_{1}$ and $U E_{2}$, selected from $\left\{A_{i}\right\}$ and $\left\{B_{j}\right\}$, respectively, to perform NOMA, as represented in Fig. 1.

The proposed cooperative NOMA system contains two phases referred to as direct and cooperative transmission. We assume the same time duration for the two phases $[6,44]$. In Fig. 2 the two phases are represented, where power-switching and energy-harvesting methods, exploiting the whole time slot for both energy and data transmission are considered [42]. This makes the proposed scenario distinct from the cooperative NOMA algorithms previously proposed in the literature, which are based on time switching for the energy harvesting [45-47]. Although time switching has convenient hardware implementation, it fully dedicates one time slot for energy transmission which is a disadvantage from the throughput efficiency point of view [48]. In the proposed scenario, the simultaneous data and energy transfer without dedicated time slot is considered, leading to a higher spectral efficient scenario.

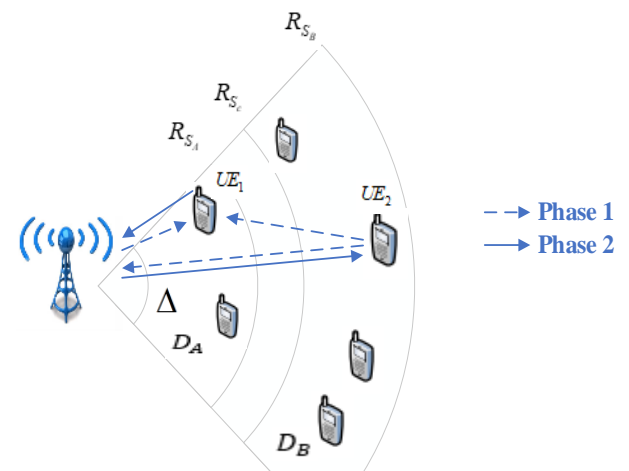

Fig. 1. Proposed cooperative mmWave-NOMA WPT uplink transmission scheme in SBS.

\begin{tabular}{|c|c|}
\hline $\begin{array}{l}\text { - Energy harvesting at UE1 } \\
\text { - Information transmission } \\
\text { from UE2 to SBS and UE1 }\end{array}$ & $\begin{array}{l}\text { - Energy harvesting at UE2 } \\
\text { - Information transmission } \\
\text { from UE1 to SBS }\end{array}$ \\
\hline
\end{tabular}

Fig. 2. Proposed process for energy harvesting and information transmission in cooperative NOMA.

\section{A. Phase 1: direct transmission}

During the first time slot, $U E_{2}$ broadcasts its signal $\left(x_{2}\right)$ where $E\left\{\left|x_{2}\right|^{2}\right\}=P_{2}$. Meanwhile, SBS can transmit wireless energy to $U E_{1}$. During this phase, $U E_{1}$ receives the message signal $x_{2}$ from $U E_{2}$ and the wireless energy signal $s$ from SBS and then combines them. At the same time, SBS receives the message signal $x_{2}$. The received signal by $\operatorname{SBS}\left(y_{B S, 1}\right)$ and $U E_{1}\left(y_{U E_{1}}\right)$ are expressed, respectively, as follows [49]:

$$
\begin{gathered}
y_{B S, 1}=h_{20} x_{2}+n_{B S, 1} \\
y_{U E_{1}}=h_{21} x_{2}+h_{01} s+n_{1}
\end{gathered}
$$

where $h_{20}$ is the channel gain between $U E_{2}$ and SBS, $h_{21}$ denotes the channel gain between $U E_{2}$ and $U E_{1}, h_{01}$ is the channel gain between SBS and $U E_{1}$, and $n_{1}$ and $n_{B S, 1}$ denote the Gaussian noise at $U E_{1}$ and SBS receiver side with variance 
$N_{0}$ and zero mean, respectively. As mentioned, $s$ is the energy signal with $E\left\{|s|^{2}\right\}=P_{s}$, assumed as deterministic and known by $U E_{1}$ [50]. According to [16], the total amount of harvested power by $U E_{1}$ is:

$$
P_{S_{1}}=\frac{\bar{P}_{H} \cdot\left(e^{w_{1} P_{S}\left|h_{01}\right|^{2}}-1\right)}{e^{w_{1} P_{S}\left|h_{01}\right|^{2}}+e^{w_{1} w_{2}}}
$$

where $\bar{P}_{H}$ represents the maximum harvested power when the energy harvesting circuit is saturated, and $w_{1}$ and $w_{2}$ are two constants modelling the $\mathrm{EH}$ circuit specifications, such as diode turn-on voltage capacitance and resistance.

\section{B. Phase 2: cooperative transmission}

During this phase, the SBS transmits energy to $U E_{2}$; similar to the first phase, the total amount of harvested power by $U E_{2}$ is:

$$
P_{S_{2}}=\frac{\bar{P}_{H} \cdot\left(e^{w_{1} P_{S}\left|h_{02}\right|^{2}}-1\right)}{e^{w_{1} P_{S}\left|h_{02}\right|^{2}}+e^{w_{1} w_{2}}}
$$

where $h_{02}$ denotes the channel gain between SBS and $U E_{2}$. At the same time, $U E_{1}$ sends the superimposed signal $x_{2}^{\prime}+x_{1}$ to SBS, where $x_{1}$ is the message signal of $U E_{1}, E\left\{\left|x_{1}\right|^{2}\right\}=P_{1}$, and $x_{2}^{\prime}$ denotes the $U E_{2}$ message signal, $E\left\{\left|x_{2}^{\prime}\right|^{2}\right\}=P_{2}^{\prime}$, relayed by $U E_{1}$. $U E_{1}$ utilizes its primary power which is denoted by $P_{U E}^{\min }$ and the harvested power for decoding and transmission. Hence, the received signal at the SBS receiver side in the second time slot can be expressed as:

$$
y_{B S, 2}=h_{10}\left(x_{1}+x_{2}^{\prime}\right)+n_{B S, 2}
$$

where $h_{10}$ is the channel gain between $U E_{1}$ and SBS, and $n_{B S, 2}$ denotes the received Gaussian noise of SBS with variance $N_{0}$ and zero-mean. Since the SBS combines the received signals of phase 1 and phase 2, and the conjugates of $h_{10}$ and $h_{20}$, by using the maximum ratio combining (MRC) method [51], it is possible to write the received signal at the SBS side as:

$$
y=\frac{h_{10}^{*}}{\left|h_{10}^{*}\right|} y_{B S, 2}+\frac{h_{20}^{*}}{\left|h_{20}^{*}\right|} y_{B S, 1}
$$

At the SBS receiver side, SIC is applied for detecting the messages [52]. According to NOMA in the uplink mode, the SBS first decodes the highest channel gain users' messages; thus, the highest channel gain user receives interference from the other users [52]. Then, the lowest channel gain user enjoys no interference.

Therefore, signal to interference plus noise ratios (SINRs) for $x_{1}$ and $x_{2}$ are calculated based on the highest channel gain of users as follows:

1) When the sum of $U E_{2}$ signals is the strongest, i.e.,

$$
\left|h_{10}\right|^{2} x_{2}^{\prime}+\left|h_{20}\right|^{2} x_{2}>\left|h_{10}\right|^{2} x_{1}
$$

In this case the signal of $U E_{2}$ is the strongest, hence the SINR of $x_{2}\left(\gamma_{U E_{2}}\right)$ and the SINR of $x_{1}\left(\gamma_{U E_{1}}\right)$ are respectively formulated as:

$$
\begin{gathered}
\gamma_{U E_{2}}=\frac{\left|h_{10}\right|^{2} P_{2}^{\prime}+\left|h_{20}\right|^{2} P_{2}}{P_{1}\left|h_{10}\right|^{2}+N_{0}} \\
\gamma_{U E_{1}}=\frac{P_{1}\left|h_{10}\right|^{2}}{N_{0}}
\end{gathered}
$$

2) When the UE $E_{1}$ signal is the strongest, i.e.,

$$
\left|h_{10}\right|^{2} x_{2}^{\prime}+\left|h_{20}\right|^{2} x_{2}<\left|h_{10}\right|^{2} x_{1}
$$

In this case, the SINR of $x_{1}\left(\gamma_{U E_{1}}\right)$ and the SINR of $x_{2}\left(\gamma_{U E_{2}}\right)$ are obtained respectively as:

$$
\begin{gathered}
\gamma_{U E_{1}}=\frac{P_{1}\left|h_{10}\right|^{2}}{\left|h_{10}\right|^{2} P_{2}^{\prime}+\left|h_{20}\right|^{2} P_{2}+N_{0}} \\
\gamma_{U E_{2}}=\frac{\left|h_{10}\right|^{2} P_{2}^{\prime}+\left|h_{20}\right|^{2} P_{2}}{N_{0}}
\end{gathered}
$$

The achievable data rate of each user at the SBS for $U E_{1}$ $\left(R_{S C U_{1}}^{x_{1}}\right)$ and $U E_{2}\left(R_{S C U_{2}}^{x_{2}}\right)$ can be calculated as

$$
\begin{aligned}
& R_{U E_{1}}=\frac{1}{2} \log _{2}\left(1+\gamma_{U E_{1}}\right) \\
& R_{U E_{2}}=\frac{1}{2} \log _{2}\left(1+\gamma_{U E_{2}}\right)
\end{aligned}
$$

and the overall $E E$ can be expressed as

$$
E E=\frac{R_{U E_{1}}+R_{U E_{2}}}{P_{1}+P_{2}+P_{2}^{\prime}+P_{c i r}}
$$

where $P_{\text {cir }}$ denotes the circuit power consumption [30, 53] which is assumed the same for both $U E_{1}$ and $U E_{2}$.

\section{Channel model}

In this paper, we introduce a new cooperative mmWaveNOMA uplink transmission scheme for SCUs where each SBS is equipped with $M$ antennas and each user has single antenna. Moreover, as explained before, each SBS supports two groups of users as $\left\{A_{i}\right\}$ and $\left\{B_{j}\right\}$. SCUs and the SBS communicate through mmWave channel, which contains a line-of-sight (LOS) path and several non-line-of-sight (NLOS) paths as:

$$
\begin{gathered}
h_{k}=\sqrt{M} \frac{\beta_{k, L} a\left(\theta_{k, 0}\right)}{\sqrt{1+d_{k}^{\alpha_{L}}}}+\sqrt{M} \sum_{l=1}^{L} \frac{\beta_{k, N L} a\left(\theta_{k, l}\right)}{\sqrt{1+d_{k}^{\alpha_{N L}}}} \\
a(\theta)=\frac{1}{\sqrt{M}}\left[1, e^{-j \pi \theta}, \ldots, e^{-j \pi(M-1) \theta}\right]^{T}
\end{gathered}
$$

where $M$ is the number of transmit antennas of SBS, $d_{k}$ is the distance between the SBS and the $k$-th SCU, $\beta_{k, L}$ and $\beta_{k, N L}$ denote the complex gains for LOS and NLOS paths, respectively, $\theta_{k, l}$ represents normalized direction of each path, $\alpha_{L}$ and $\alpha_{N L}$ indicate the path loss exponents for the LOS and NLOS paths, respectively, and $L$ shows the number of multipaths [25]. Since LOS path is dominant [25], mmWave channel can be modelled as

$$
h_{k}=\sqrt{M} \frac{\beta_{k} a\left(\theta_{k}\right)}{\sqrt{1+d_{k}^{\alpha_{L}}}}
$$

As discussed in [25], to reduce system overhead, random beamforming is considered in SBS. The beam is described as:

$$
Q=a(v)
$$

where $v$ is a random variable uniformly distributed in the range [-11]. Similar to [22], the effective channel gain of the $k$-th $\mathrm{SCU}$ is formulated as: 


$$
\begin{aligned}
\left|h_{k}^{H} Q\right|^{2} & =\frac{M\left|\beta_{k}\right|^{2}\left|a\left(\theta_{k}\right)^{H} Q\right|^{2}}{1+d_{k}^{\alpha}} \\
& =\frac{\left|\beta_{k}\right|^{2}\left|\sum_{n=0}^{M-1} e^{j \pi n\left(v-\theta_{k}\right)}\right|^{2}}{M\left(1+d_{k}^{\alpha}\right)} \\
& =\frac{\left|\beta_{k}\right|^{2} \sin ^{2}\left(\frac{\pi M\left(v-\theta_{k}\right)}{2}\right)}{M\left(1+d_{k}^{\alpha}\right) \sin ^{2}\left(\frac{\pi\left(v-\theta_{k}\right)}{2}\right)} \\
& =\frac{\left|\beta_{k}\right|^{2}}{\left(1+d_{k}^{\alpha}\right)} F_{M}\left(v-\theta_{k}\right)
\end{aligned}
$$

where $F_{M}(\cdot)$ denotes the Fejér kernel defined as:

$$
F_{M}\left(v-\theta_{k}\right)=\frac{\sin ^{2}\left(\frac{\pi M\left(v-\theta_{k}\right)}{2}\right)}{M \sin ^{2}\left(\frac{\pi\left(v-\theta_{k}\right)}{2}\right)} .
$$

\section{PRoposed EE METHOD}

In this paper, we investigate the joint user grouping and power allocation problem in cooperative mmWave NOMA systems. The power allocated to the users are denoted as $P=$ $\left\{P_{1}^{*}, P_{2}^{*}, P_{2}^{\prime *}\right\}$ and the grouped users are denoted as $U=\left\{U E_{1}\right.$, $\left.U E_{2}\right\}$. The main objective is to maximize the $\mathrm{EE}$ of system subject to the constraints on the minimum data rate of each user and total power as follows:

$$
\begin{gathered}
\qquad\left(U^{*}, P^{*}\right)=\underset{U, P}{\operatorname{argmax}} E E \\
\text { subject to } R_{U E_{1}} \geq R_{U E_{1}}^{\min } \\
R_{U E_{2}} \geq R_{U E_{2}}^{\text {min }} \\
0 \leq P_{1}+P_{2}^{\prime} \leq P_{1}^{\max } \\
0 \leq P_{2} \leq P_{2}^{\max }
\end{gathered}
$$

where $U^{*}$ denotes the optimum user grouping scheme and $P^{*}$ contains the optimum power of $U E_{1}$ and the optimum powers of $U E_{2}$ in direct and cooperative modes, respectively. $R_{U E_{1}}^{\min }$ and $R_{U E_{2}}^{\min }$ represent the minimum required data rate constraints of $U E_{1}$ and $U E_{2}$, respectively, and $P_{1}^{\max }$ is the maximum transmitted power of $U E_{1}$ evaluated as $P_{1}^{\max }=P_{S_{1}}+P_{U E}^{\min }-$ $P_{C}$, where $P_{U E}^{\min }$ is the primary power of $U E_{1}, P_{S_{1}}$ is the harvested power during the direct transmission phase and $P_{C}$ denotes the considered constant power for the decoding. Finally, $P_{2}^{\max }$ is the maximum transmitted power of $U E_{2}$ evaluated as $P_{2}^{\max }=P_{S_{2}}+P_{U E}^{\min }$. Similarly, $P_{U E}^{\min }$ is the primary power of $U E_{2}$, and $P_{S_{2}}$ shows the harvested power during the cooperative transmission phase.

Since the EE in (13) is non-convex [54], the maximization problem in (19) will be NP-hard and non-convex. Hence, for more simplicity, the problem can be decoupled into two independent sub-problems of user grouping and power allocation, which are explained in the following.

\section{A. User Grouping Scheme}

As shown in Fig. 1, the SBS generates randomly a beamforming vector. Only uniformly distributed users that fall into the angle $2 \Delta$ will be scheduled to ensure the maximal angle difference $\Delta$ between selected users and their associated beam. Thus, it is not required to know all the nodes, while only those falling in the specified angle. This significantly reduces the system overhead in an ultra-dense network. Moreover, since the mmWave transmission is highly directional, in the proposed NOMA grouping algorithm, we avoid scheduling those users who may have low signal strength, which enhances the implementation of WPT, reduces the search between all users and, thus, reduces system overhead.

The optimal user grouping scheme, when implemented for NOMA uplink, can be seen as a discrete problem, and solved by searching over all existing pairs of users. Therefore, to reduce the computational complexity we propose intuitive algorithms for user grouping which are described in the following:

1) Weakest far user with optimum near user (WFON): the user with the lowest channel gain among the far users $\left\{B_{j}\right\}$ is selected to provide high channel gain difference between paired users, which is desired for NOMA implementation and reduces the number of iterations to search over the far users. Then, for each pair of near users and selected far user, the optimal transmit powers are calculated based on Algorithm 2 and their EEs are obtained according to (13). Then, to select a near user among $\left\{A_{i}\right\}$, the user that provides the maximum EE will be selected as the second node for uplink NOMA implementation. This scheme is described in more details in Algorithm 1.

2) Weakest far user with sub-optimum near user (WFSN): similar to the previous case, first the user with the lowest channel gain among the far users $\left\{B_{j}\right\}$ is selected. Then, for further reducing the complexity, a user grouping solution based on the fixed power allocation is proposed. With the observation of the NOMA principle that the power allocation of far users should be more than near users $[6,55]$, the power of users is assumed as $P_{1}=$ $P_{2}^{\prime}=P_{1}^{\max } / 4$ and $P_{2}=P_{2}^{\max }$, respectively. Then, the EEs for each pair of near users and selected far user are obtained according to (13). Finally, to select a near user among $\left\{A_{i}\right\}$, the user that provides the maximum EE will be selected as the second node for uplink NOMA implementation. The pseudo-code of this algorithm is similar to Algorithm 1, excluding the EE calculation step.

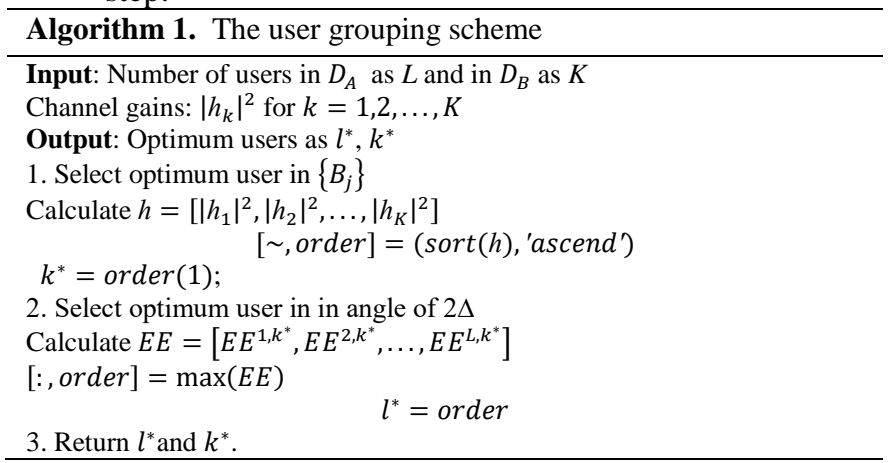

\section{B. Proposed Power Allocation Method}

We propose a method exploiting the BA approach for solving the EE problem. The BA is a meta-heuristic algorithm, inspired 
by the echolocation properties of bats. The advantage of BA over the existing optimization algorithms is its flexibility, simplicity, and low programming effort [56]. Other metaheuristic algorithms such as particle swarm optimization (PSO) and harmony search (HS), can be considered as a simplified version of BA where BA performs better than other methods in finding the optimal solution [57].

The EE optimization problem in (19) is a constrained optimization problem. To implement BA, we introduce a penalization function. The goal is to transform the constrained problem (19) into an unconstrained problem. Note that, in the proposed EE optimization problem, the third and fourth terms are constraints on the powers, considered as the positions of the bats in Algorithm 2. In the updating stage of BA, the generated position is ensured by the boundaries of the optimization parameters which are mentioned in the third and fourth constraint of (19). If the generated position exceeds the boundaries, it would be limited to the boundary value. Therefore, the problem is transformed into a maximization problem as follows:

$$
f(x)=E E-\delta \cdot \operatorname{Res}(x)
$$

where $\delta$ denotes the penalizing factor, and the penalty function is defined as:

$$
\begin{aligned}
\operatorname{Res}(x)=\min \{0 & \left., R_{U E_{1}}^{x_{1}}(x)-R_{U E_{1}}^{\min }\right\}^{2} \\
& +\min \left\{0, R_{U E_{2}}^{x_{2}}(x)-R_{U E_{2}}^{\min }\right\}^{2}
\end{aligned}
$$

The pseudo code of a generic optimization problem implemented trough the BA is given in Algorithm 2.

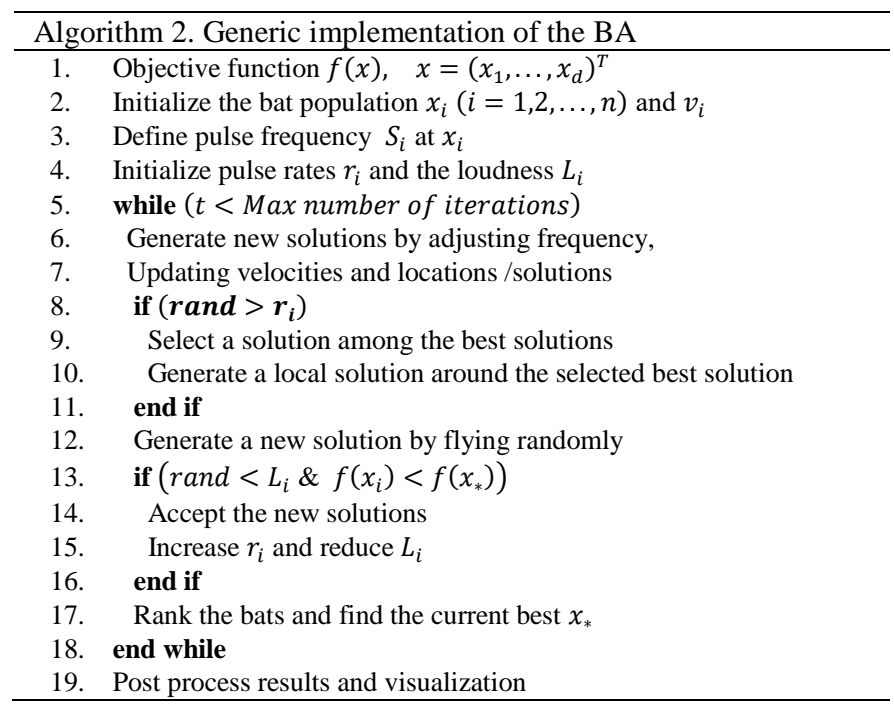

The main steps for EE optimization based on BA are explained as follow:

- $\quad$ The three parameters in the optimization problem, $P_{1}$, $P_{2}$ and $P_{2}^{\prime}$, are considered as the positions of the bats, i.e., $x$ in Algorithm 1.

- The boundaries of optimization parameters are $P_{1}^{\max }$ and $P_{2}^{\max }$ as described in (19) which are affected by the harvested energy from mmWave WPT.

- The number of solutions in the search space is described by the population size $b$, which is set to 40 .
- The generation size $(G)$ corresponds to the number of iterations in the BA, allowing to find the best result. Here we set $G$ to 20 [58].

- The frequency $\left(S_{b, k+1}\right)$ of current bat $(b)$ at time step $k+1$, is updated as

$$
S_{b, k+1}=S_{\text {min }}+\left(S_{\max }-S_{\min }\right) \xi
$$

where $\xi$ is a random variable uniformly distributed in the range $[0,1]$, and $S_{\min }$ and $S_{\max }$ represent the minimum and maximum values of frequency for the current bat $(b)$, which are set to 0 and 1 , respectively.

- The velocity $\left(V_{b, k+1}\right)$ of current bat at time step $k+1$ is updated with the following rule

$$
V_{b, k+1}=V_{b, k}+\left(x_{b, k}-x^{*}\right) S_{b, k+1}
$$

where $V_{b, k}$ is the velocity of current bat at time step $k$, $x_{b, k}$ is the position of current bat at time step $k$, and $x^{*}$ defines the best global solution.

- $x_{b, k+1}$ defines the position of current bat at the time step $k+1$ and is formulated as

$$
x_{b, k+1}=x_{b, k}+v_{b, k+1}
$$

- Local solution is defined around the best solution as

$$
x_{n e w}=x_{\text {old }}+\xi L_{k}
$$

where $\xi$ randomly lies in the range $[-1,1]$, and $L_{k}$ denotes the average loudness of bats at the time step $k$.

- $\quad$ As the bat approaches the target, the loudness $\left(L_{b, k+1}\right)$ and pulse rate $\left(r_{b, k+1}\right)$ are respectively updated as:

$$
\begin{gathered}
L_{b, k+1}=\alpha L_{b, k} \\
r_{b, k+1}=r_{b, 0}\left(1-e^{-\sigma k}\right)
\end{gathered}
$$

where $\alpha$ and $\sigma$ are constant values which are set to $\alpha=$ $\sigma=0.9$ [57]. Initial loudness $\left(L_{b, 0}\right)$ and initial pulse rate $\left(r_{b, 0}\right)$ are selected randomly between $[1,2]$ and $[0$, 1], respectively [59].

- The best solution $\left(x^{*}\right)$ is updated.

\section{THEORETICAL ANALYSIS}

In this section, we derive the exact analytical expressions for the energy outage probability of the WPT and the outage probability of two users to show the efficiency of mmWave WPT and the proposed NOMA cooperative method. These metrics are discussed in detail in the following.

\section{A. Energy outage probability}

We consider the non-linear model in [16] for the energy harvesting. Following (3) and (4), the amount of harvested energy by each user can be written as

$$
E_{s_{i}}=T \frac{\bar{P}_{H} \cdot\left(e^{w_{1} P_{S}\left|h_{i}\right|^{2}}-1\right)}{e^{w_{1} P_{S}\left|h_{i}\right|^{2}}+e^{w_{1} w_{2}}}
$$

where $i \in\{1,2\}$ is the user ID. In practical situations, the amount of harvested energy from the SBS has to be higher than a predefined threshold that we denote as $e_{t h}$. Therefore, in the following it is possible to refer to the energy outage probability of $U E_{1}$ and $U E_{2}$ as the probability that the users' harvested 
energy is lower than $e_{t h}$. Mathematically, the energy outage probabilities of $U E_{1}$ and $U E_{2}$ can be written as $P\left(E_{s_{1}}<e_{t h}\right)$ and $P\left(E_{S_{2}}<e_{t h}\right)$, respectively. The energy outage probability of $U E_{1}$ is related to the link between users and SBS. Hence, as described in (18), the probability density function of the effective channel gain for the mmWave link can be written as:

$$
f_{\left|h_{k}^{H} Q\right|}(x)=\frac{\left(1+d_{k}^{\alpha}\right)}{F_{M}\left(v-\theta_{k}\right)} e^{-x \frac{\left(1+d_{k}^{\alpha}\right)}{F_{M}\left(v-\theta_{k}\right)}}
$$

and the energy outage probability of $U E_{1}$ is formulated as [60]:

$$
\begin{aligned}
& P_{U E_{1}}^{e}=P\left(\left|h_{01}\right|^{2}<\frac{\ln \left(\frac{1+\frac{e^{t h}}{T \cdot \bar{P}_{H}} e^{w_{1} w_{2}}}{1-\frac{e^{t h}}{T \cdot \bar{P}_{H}}}\right)}{w_{1} P_{S}}\right) \\
& =\frac{\left(1+d_{U E_{1}}^{\alpha}\right)}{F_{M}\left(v-\theta_{U E_{1}}\right)} \int_{0}^{\ln \left(\frac{1+\frac{e^{t h}}{T \cdot \bar{P}_{H}} e^{w_{1} w_{2}}}{1-\frac{e^{t h}}{T \cdot \bar{P}_{H}}}\right)} e^{-x \frac{\left(1+d_{U E_{1}}^{\alpha}\right)}{w_{1} P_{S}}} \\
& =1-e^{-\frac{\ln \left(\frac{1+\frac{e^{t h}}{T \cdot \bar{P}_{H}} w_{1} w_{1}}{1-\frac{e^{t h}}{T \cdot \bar{P}_{H}}}\right)}{w_{1} P_{S}} F_{\left(1+d_{U E_{1}}^{\alpha}\right)}^{\alpha\left(v-\theta_{U E_{1}}\right)}}
\end{aligned}
$$

For the special case when $e_{t h} \rightarrow 0$, (30) can be approximated as:

$$
P_{U E_{1}}^{e} \approx \frac{\ln \left(\frac{1+\frac{e^{t h}}{T \cdot \bar{P}_{H}} e^{w_{1} w_{2}}}{1-\frac{e^{t h}}{T \cdot \bar{P}_{H}}}\right)}{w_{1} P_{S}} \frac{\left(1+d_{U E_{1}}^{\alpha}\right)}{F_{M}\left(v-\theta_{U E_{1}}\right)}
$$

that is obtained by considering $e^{-y}=1-y$ for $y \rightarrow 0$ [60]. Noting that, $\left|v-\theta_{U E_{1}}\right| \leq \Delta$, for $\Delta \rightarrow 0$ the Fejér kernel can be approximated as [60]:

$$
F_{M}\left(v-\theta_{U E_{1}}\right) \approx M\left(1-\frac{\pi^{2} M^{2}\left(v-\theta_{U E_{1}}\right)^{2}}{12}\right)
$$

Moreover, by using $(1-x)^{-1} \approx(1+x)$ for $x \rightarrow 0,(31)$ is approximated as [60]:

$$
\begin{aligned}
& P_{U E_{1}}^{e} \approx \frac{\ln \left(\frac{1+\frac{e^{t h}}{T \cdot \bar{P}_{H}} e^{w_{1} w_{2}}}{1-\frac{e^{t h}}{T \cdot \bar{P}_{H}}}\right)}{M w_{1} P_{S}}(1 \\
& \left.+\frac{\pi^{2} M^{2}\left(v-\theta_{U E_{1}}\right)^{2}}{12}\right)\left(1+d_{U E_{1}}^{\alpha}\right)
\end{aligned}
$$

Using similar steps, the energy outage probability of $U E_{2}$ is obtained as:

$$
\begin{gathered}
P_{U E_{2}}^{e}=1-e^{-\frac{\ln \left(\frac{1+\frac{e^{t h}}{T \cdot \bar{P}_{H}} e^{w_{1} w_{2}}}{1-\frac{e^{t h}}{T \cdot \bar{P}_{H}}}\right)}{M w_{1} P_{S}} F_{M}\left(1+d_{U E_{2}}^{\alpha}\right)} \\
\approx \frac{\ln \left(\frac{1+\frac{e^{t h}}{\left.T \cdot \bar{P}_{H}\right)} e^{w_{1} w_{2}}}{1-\frac{e^{t h}}{T \cdot \bar{P}_{H}}}\right)}{M w_{1} P_{S}}(1 \\
\left.+\frac{\pi^{2} M^{2}\left(v-\theta_{U E_{2}}\right)^{2}}{12}\right)(1 \\
\left.+d_{U E_{2}}^{\alpha}\right)
\end{gathered}
$$

Therefore, the energy outage probability of users is small for small distances between the users and SBS. Moreover, for small values of $\left|v-\theta_{U E_{1}}\right|$, the energy outage probability decreases by increasing the number of transmit antennas $M$.

\section{B. Outage probability}

The outage probability of users in NOMA-based SBS occurs when the BS cannot decode the signals of $U E_{1}$ and $U E_{2}$ successfully. Thus, to have successful decoding of signals, the target data rates for $U E_{1}$ and $U E_{2}$ are defined as $R_{1}$ and $R_{2}$, respectively, which lead to $\varepsilon_{1}=2^{2 R_{1}}-1$ and $\varepsilon_{2}=2^{2 R_{2}}-1$. Thus, the outage probabilities of $U E_{1}$ and $U E_{2}$ for the two cases in the NOMA system are formulated in the following. 1) When the sum of $U E_{2}$ signals is the strongest, i.e., $\left|h_{10}\right|^{2} x_{2}^{\prime}+\left|h_{20}\right|^{2} x_{2}>\left|h_{10}\right|^{2} x_{1}$.

The outage probability of $U E_{2}\left(P_{U E_{2}}^{O}\right)$ in this case can be expressed as [61]:

$$
P_{U E_{2}}^{O}=1-P\left(\gamma_{U E_{2}}>\varepsilon_{2}\right)
$$

then the outage probability of $U E_{1}\left(P_{U E_{1}}^{O}\right)$ is also obtained as [61]:

$$
P_{U E_{1}}^{O}=1-P\left(\gamma_{U E_{1}}>\varepsilon_{1}, \gamma_{U E_{2}}>\varepsilon_{2}\right)
$$

a)

\section{Outage Probability of $U E_{2}$}

The outage probability of $U E_{2}$ is related to the link between users and SBS. Hence, by using (18), the outage probability of $U E_{2}$ can be written as (37).

\section{b) Outage Probability of $U E_{1}$}

Exploiting (36), the outage probability of $U E_{1}$ can be formulated as in (38)

2) When $U E_{1}$ signal is the strongest, i.e., $\left|h_{10}\right|^{2} x_{2}^{\prime}+$ $\left|h_{20}\right|^{2} x_{2}<\left|h_{10}\right|^{2} x_{1}$ :

The outage probability of $U E_{1}\left(P_{U E_{1}}^{O}\right)$ in this case can be obtained as [61]:

$$
P_{U E_{1}}^{O}=1-P\left(\gamma_{U E_{1}}>\varepsilon_{1}\right)
$$

The outage probability of $U E_{2}\left(P_{U E_{2}}^{O}\right)$ is then expressed as [61]:

$$
P_{U E_{2}}^{O}=1-P\left(\gamma_{U E_{1}}>\varepsilon_{1}, \gamma_{U E_{2}}>\varepsilon_{2}\right)
$$

\section{a) Outage Probability of $U E_{1}$}

The outage probability of $U E_{1}$ can be calculated in a similar way considering (7), as shown in (41). 


$$
\begin{aligned}
& P_{U E_{2}}^{O}=1-P\left(\left|h_{20}\right|^{2}>\frac{\left|h_{10}\right|^{2}\left(\varepsilon_{2} P_{1}-P_{2}^{\prime}\right)+\varepsilon_{2} N_{0}}{P_{2}}\right) \\
& =1-\frac{\left(1+d_{U E_{1}}^{\alpha}\right)}{F_{M}\left(v-\theta_{U E_{1}}\right)} \int_{0}^{\infty} e^{-\frac{\left(x\left(\varepsilon_{2} P_{1}-P_{2}^{\prime}\right)+\varepsilon_{2} N_{0}\right)\left(1+d_{U E_{2}}^{\alpha}\right)}{P_{2} F_{M}\left(v-\theta_{U E_{2}}\right)}} e^{-x \frac{\left(1+d_{U E_{1}}^{\alpha}\right)}{F_{M}\left(v-\theta_{U E_{1}}\right)}} d x \\
& =\frac{\left(\varepsilon_{2} P_{1}-P_{2}^{\prime}\right) F_{M}\left(v-\theta_{U E_{1}}\right)\left(1+d_{U E_{2}}^{\alpha}\right)+P_{2} F_{M}\left(v-\theta_{U E_{2}}\right)\left(1+d_{U E_{1}}^{\alpha}\right)\left(1-e^{-\frac{\left(\varepsilon_{2} N_{0}\right)\left(1+d_{U E_{2}}^{\alpha}\right)}{P_{2} F_{M}\left(v-\theta_{U E_{2}}\right)}}\right)}{\left(\varepsilon_{2} P_{1}-P_{2}^{\prime}\right) F_{M}\left(v-\theta_{U E_{1}}\right)\left(1+d_{U E_{2}}^{\alpha}\right)+P_{2} F_{M}\left(v-\theta_{U E_{2}}\right)\left(1+d_{U E_{1}}^{\alpha}\right)} \\
& P_{U E_{1}}^{O}=1-P\left(\left|h_{10}\right|^{2}>\frac{\varepsilon_{1} N_{0}}{P_{1}},\left|h_{20}\right|^{2}>\frac{\left|h_{10}\right|^{2}\left(\varepsilon_{2} P_{1}-P_{2}^{\prime}\right)+\varepsilon_{2} N_{0}}{P_{2}}\right) \\
& =1-\frac{\left(1+d_{U E_{1}}^{\alpha}\right)}{F_{M}\left(v-\theta_{U E_{1}}\right)} \int_{\frac{\varepsilon_{1} N_{0}}{P_{1}}}^{\infty} e^{-\frac{\left(x\left(\varepsilon_{2} P_{1}-P_{2}^{\prime}\right)+\varepsilon_{2} N_{0}\right)\left(1+d_{U E_{2}}^{\alpha}\right)}{P_{2} F_{M}\left(v-\theta_{U E_{2}}\right)}} e^{-x \frac{\left(1+d_{U E_{1}}^{\alpha}\right)}{F_{M}\left(v-\theta_{U E_{1}}\right)}} d x \\
& =1-\frac{\frac{\left(1+d_{U E_{1}}^{\alpha}\right)}{F_{M}\left(v-\theta_{U E_{1}}\right)} e^{-\frac{\left(\varepsilon_{2} N_{0}\right)\left(1+d_{U E_{2}}^{\alpha}\right)}{P_{2} F_{M}\left(v-\theta_{U E_{2}}\right)}}}{\frac{\left(1+d_{U E_{1}}^{\alpha}\right)}{F_{M}\left(v-\theta_{U E_{1}}\right)}+\frac{\left(\varepsilon_{2} P_{1}-P_{2}^{\prime}\right)\left(1+d_{U E_{2}}^{\alpha}\right)}{P_{2} F_{M}\left(v-\theta_{U E_{2}}\right)}} e^{-\frac{\varepsilon_{1} N_{0}}{P_{1}}\left(\frac{\left(1+d_{U E_{1}}^{\alpha}\right)}{F_{M}\left(v-\theta_{U E_{1}}\right)}+\frac{\left(\varepsilon_{2} P_{1}-P_{2}^{\prime}\right)\left(1+d_{U E_{2}}\right)}{P_{2} F_{M}\left(v-\theta_{U E_{2}}\right)}\right)}
\end{aligned}
$$

\section{b) Outage Probability of $U E_{2}$}

Similarly, the outage probability of $U E_{2}$ is formulated as in (42) where $\eta_{U E_{2}}=\max \left\{\frac{N_{0} \varepsilon_{2}-\left|h_{2}\right|^{2} P_{2}}{P_{2}^{\prime}}, \frac{\varepsilon_{1}\left|h_{20}\right|^{2} P_{2}+N_{0} \varepsilon_{1}}{\left(P_{1}-\varepsilon_{1} P_{2}^{\prime}\right)}\right\}$. Although (42) involves an infinite integral, it can be solved using computational software such as "Mathematica".

By comparing the outage probability of $U E_{1}$ and $U E_{2}$ in the two cases, it can be seen how the transmitted signal in cooperative phase impacts the outage probabilities of $U E_{1}$ and $U E_{2}$. In the first case, the outage probability of $U E_{2}$ exceeds that of $U E_{1}$. In the second case, by comparing the integration intervals, it can be observed that the outage probability of $U E_{1}$ is greater or at least equal to $U E_{2}$.

\section{Diversity Order Analysis}

Here, to gain deeper insights for cooperative network, based on the derived outage probabilities at high SNR regimes the diversity order of the proposed approach is obtained:

$$
d_{i}=-\lim _{\rho \rightarrow \infty} \frac{\log \left(P_{U E_{i}}^{O}(\rho)\right)}{\log (\rho)}
$$

where $\rho=\frac{P_{1}}{N_{0}}=\frac{P_{2}^{\prime}}{N_{0}}=\frac{P_{2}}{2 N_{0}}$, and $P_{U E_{i}}^{O}$ denotes the outage probability of users, with $i=1,2$. Considering (43) and the analytical results in (37)-(42), the diversity order of $U E_{1}$ and $U E_{2}$ for the two cases with $e^{-y}=1-y$ for $y \rightarrow 0$, are formulated as follows.

1) When the sum of $U E_{2}$ signals is the strongest, i.e., $\left|h_{10}\right|^{2} x_{2}^{\prime}+\left|h_{20}\right|^{2} x_{2}>\left|h_{10}\right|^{2} x_{1}$.

After mathematical simplifications, (37) and (38) can be formulated as (44) and (45). Then, for $\rho \rightarrow \infty$ and $\varepsilon_{2}, \varepsilon_{1}=1$ the diversity orders of $U E_{2}$ and $U E_{1}$ can be defined as:

$$
d_{1}=1, d_{2}=1
$$

Proof: By using (44) and (45) and substituting them into (43) and ignoring the higher order of $\rho$ in the high SNR regime, the diversity orders equal to one can be obtained straightforwardly. This completes the proof.

Remark 1 . Note that, differently from the conclusions that the diversity orders of uplink NOMA systems are zero due to impact of residual interference [62-65], the diversity order of the proposed cooperative network is 1 . This is due to the fact that cooperation enhances the received signal of the far user and enhances the diversity.

2) When $U E_{1}$ signal is the strongest, i.e., $\left|h_{10}\right|^{2} x_{2}^{\prime}+$ $\left|h_{20}\right|^{2} x_{2}<\left|h_{10}\right|^{2} x_{1}$ :

Similar to the previous case, (41) is formulated as (46). Then, for $\rho \rightarrow \infty$ and $\varepsilon_{2}, \varepsilon_{1}=1$, the diversity order of $U E_{1}$ is obtained as follow:

$$
d_{1}=0
$$

In addition, by considering:

$$
\max \left\{\frac{N_{0} \varepsilon_{2}-\left|h_{2}\right|^{2} P_{2}}{P_{2}^{\prime}}, \frac{\varepsilon_{1}\left|h_{20}\right|^{2} P_{2}+N_{0} \varepsilon_{1}}{\left(P_{1}-\varepsilon_{1} P_{2}^{\prime}\right)}\right\}=\frac{\varepsilon_{1}\left|h_{20}\right|^{2} P_{2}+N_{0} \varepsilon_{1}}{\left(P_{1}-\varepsilon_{1} P_{2}^{\prime}\right)},
$$

since $P_{1}-\varepsilon_{1} P_{2}^{\prime}$ goes to zero, the outage probability of $U E_{2}$ and $U E_{1}$ is the same. Hence, diversity order of $U E_{2}$ is zero too.

Proof: By using (47) and substituting it into (43), the diversity orders equal to zero can be obtained straightforwardly. This completes the proof.

Remark 2. Let us note that the difference between this case and previous case is the decoding order. In this case, the signal of $U E_{1}$ is decoded first, leading to suffer from the interference from $U E_{2}$. This means that the increased power of the desired signal $U E_{1}$ is offset by interference signals of the cooperation phase since this signal also gets larger. Therefore, the diversity order of zero for the users can be solved by considering the power allocation of users based on the desired decoding order of users in the BS. 


$$
\begin{aligned}
& P_{U E_{1}}^{O}=1-P\left(\left|h_{10}\right|^{2}>\frac{\varepsilon_{1}\left|h_{20}\right|^{2} P_{2}+N_{0} \varepsilon_{1}}{\left(P_{1}-\varepsilon_{1} P_{2}^{\prime}\right)}\right)=1-\frac{\left(1+d_{U E_{2}}^{\alpha}\right)}{F_{M}\left(v-\theta_{U E_{2}}\right)} \int_{0}^{\infty} e^{-\frac{x\left(1+d_{U E_{1}}^{\alpha} \varepsilon_{1} P_{2}+N_{0} \varepsilon_{1}\left(1+d_{U E_{1}}\right)\right.}{F_{M}\left(v-\theta_{U E_{1}}\right)\left(P_{1}-\varepsilon_{1} P_{2}^{\prime}\right)}} e^{-x \frac{\left(1+d_{U E_{2}}^{\alpha}\right)}{F_{M}\left(v-\theta_{U E_{2}}\right)}} d x \\
& =1-\frac{\left(1+d_{U E_{2}}^{\alpha}\right) F_{M}\left(v-\theta_{U E_{1}}\right)\left(P_{1}-\varepsilon_{1} P_{2}^{\prime}\right) e^{-\frac{N_{0} \varepsilon_{1}\left(1+d_{U E_{1}}^{\alpha}\right)}{F_{M}\left(v-\theta_{U E_{1}}\right)\left(P_{1}-\varepsilon_{1} P_{2}^{\prime}\right)}}}{\varepsilon_{1} P_{2} F_{M}\left(v-\theta_{U E_{2}}\right)\left(1+d_{U E_{1}}^{\alpha}\right)+\left(1+d_{U E_{2}}^{\alpha}\right) F_{M}\left(v-\theta_{U E_{1}}\right)\left(P_{1}-\varepsilon_{1} P_{2}^{\prime}\right)} \\
& P_{U E_{2}}^{O}=1-P\left(\left|h_{10}\right|^{2}>\eta_{U E_{2}}\right)=1-\frac{\left(1+d_{U E_{2}}^{\alpha}\right)}{F_{M}\left(v-\theta_{U E_{2}}\right)} \int_{0}^{\infty} e^{-\frac{\eta_{U E_{2}}\left(1+d_{U E_{1}}^{\alpha}\right)}{F_{M}\left(v-\theta_{U E_{1}}\right)}} e^{-x \frac{\left(1+d_{U E_{2}}^{\alpha}\right)}{F_{M}\left(v-\theta_{U E_{2}}\right)}} d x \\
& P_{U E_{2}}^{O}=\frac{\rho\left(\varepsilon_{2}-1\right) F_{M}\left(v-\theta_{U E_{1}}\right)\left(1+d_{U E_{2}}^{\alpha}\right)+\left(1+d_{U E_{1}}^{\alpha}\right)\left(\varepsilon_{2}\right)\left(1+d_{U E_{2}}^{\alpha}\right)}{\rho\left(\left(\varepsilon_{2}-1\right) F_{M}\left(v-\theta_{U E_{1}}\right)\left(1+d_{U E_{2}}^{\alpha}\right)+F_{M}\left(v-\theta_{U E_{2}}\right)\left(1+d_{U E_{1}}^{\alpha}\right)\right)} \\
& P_{U E_{1}}^{O}=\frac{\rho\left(\varepsilon_{2}-1\right)\left(1+d_{U E_{2}}^{\alpha}\right) F_{M}\left(v-\theta_{U E_{1}}\right)+\left(1+d_{U E_{1}}^{\alpha}\right)\left(\varepsilon_{2}\right)\left(1+d_{U E_{2}}^{\alpha}\right)}{\rho\left(\left(1+d_{U E_{1}}^{\alpha}\right) F_{M}\left(v-\theta_{U E_{2}}\right)+F_{M}\left(v-\theta_{U E_{1}}\right)\left(\varepsilon_{2}-1\right)\left(1+d_{U E_{2}}^{\alpha}\right)\right)}+\frac{\varepsilon_{1}\left(1+d_{U E_{1}}^{\alpha}\right)}{\rho F_{M}\left(v-\theta_{U E_{1}}\right)} \\
& -\frac{\left(1+d_{U E_{1}}^{\alpha}\right)}{\rho^{2} F_{M}\left(v-\theta_{U E_{1}}\right)} \frac{\left(2 \varepsilon_{1} \varepsilon_{2}\right)\left(1+d_{U E_{2}}^{\alpha}\right)}{F_{M}\left(v-\theta_{U E_{2}}\right)} \\
& P_{U E_{1}}^{O}=\frac{\left(\varepsilon_{1} P_{2} F_{M}\left(v-\theta_{U E_{2}}\right)\left(1+d_{U E_{1}}^{\alpha}\right)+\left(1+d_{U E_{2}}^{\alpha}\right) N_{0} \varepsilon_{1}\left(1+d_{U E_{1}}^{\alpha}\right)\right)}{\varepsilon_{1} P_{2} F_{M}\left(v-\theta_{U E_{2}}\right)\left(1+d_{U E_{1}}^{\alpha}\right)+\left(1+d_{U E_{2}}^{\alpha}\right) F_{M}\left(v-\theta_{U E_{1}}\right)\left(P_{1}-\varepsilon_{1} P_{2}^{\prime}\right)}
\end{aligned}
$$

\section{PERformance EVAluAtion}

In this section, the efficiency of the proposed cooperative NOMA-mmWave with WPT approach is demonstrated through simulation results. To highlight the accuracy of the analytical expressions obtained in the previous section, simulation results are presented in the Sections V.A and V.B. In Section V.C, the impact of different grouping schemes on the SE and EE of the proposed system is explored. In Section V.D, the performance of the proposed system is compared with different methods. Finally, the effectiveness of BA is explored in Section V.E. The system parameters for the sake of readability are summarized in Table I [16].

\section{TABLE I SimULATIONS PARAMETERS}

\begin{tabular}{ll}
\hline \multicolumn{1}{c}{ PARAMETER } & \multicolumn{1}{c}{ VALUE } \\
\hline Radius $R_{S_{A}}$ & $10 \mathrm{~m}$ \\
Radius $R_{S_{C}}$ & $20 \mathrm{~m}$ \\
Radius $R_{S_{B}}$ & $30 \mathrm{~m}$ \\
Maximum transmitted power of SBS $\left(P_{S}\right)$ & $20 \mathrm{dBm}$ \\
$P_{U E}^{\text {min }}$ & $0 \mathrm{dBm}$ \\
Noise power & $-174 \mathrm{dBm} / \mathrm{Hz}$ \\
$\bar{P}_{H}$ & $0.024 \mathrm{~mW}$ \\
$w_{1}$ & 1500 \\
$w_{2}$ & 0.0022
\end{tabular}

\section{A. Energy outage probability of SCUS}

Fig. 3 demonstrates the energy outage probabilities of near and far SCUs versus the energy threshold $\left(e_{t h}\right)$ where Sim denotes simulation and Ana denotes analytical expressions. We can observe the following facts: i) analytical results of (30) and (31) match the simulation results well; ii) as expected, energy outage probability of users increases by increasing the energy threshold $\left(e_{t h}\right)$; iii) for low values of $e_{t h}$, analytical approximations are very tight; iv) energy outage probabilities of far users are more than those of near users.

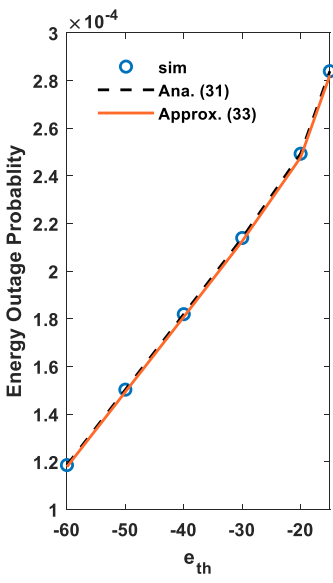

(a) near SCU

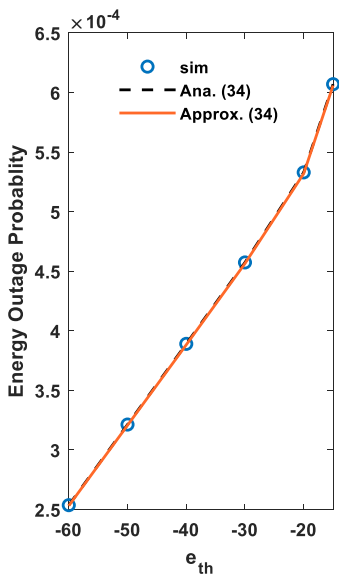

(b) far SCU
Fig. 3 Energy outage probabilities of SCU versus energy threshold $\left(e_{t h}\right)$.

Fig. 4 shows energy outage probabilities of far and near SCUs versus different number of transmit antennas for different transmit powers. As expected, higher transmit power leads to lower energy outage probability. Moreover, increasing the number of transmit antennas results in lower energy outage probability, since transmission link between SCU and SBS is improved by providing higher directive gain. 


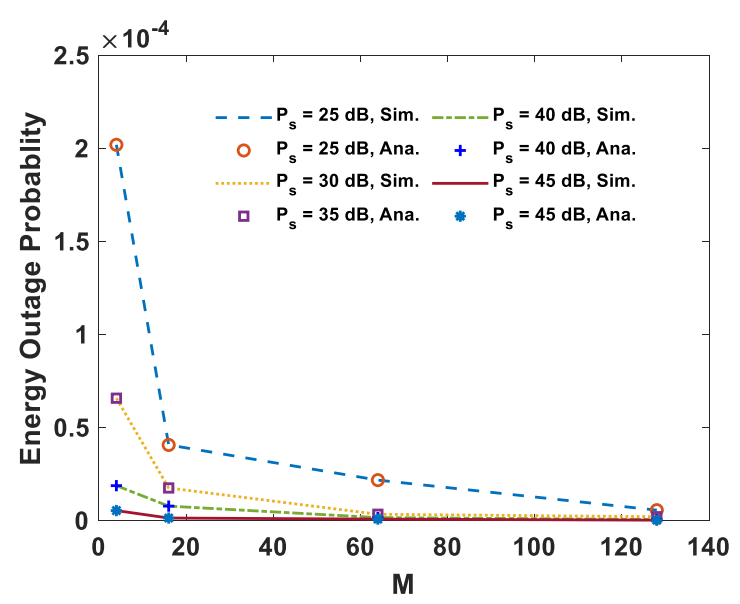

(a) near SCU

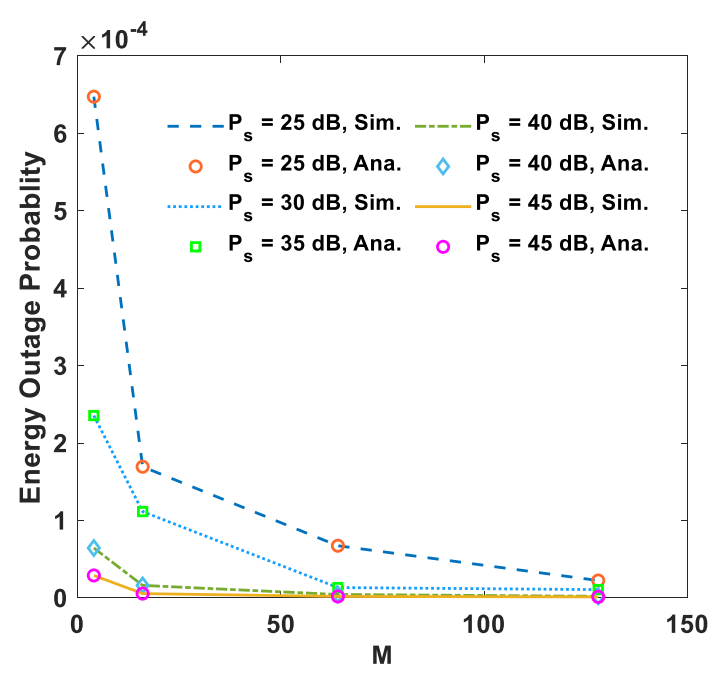

(b) far SCU

Fig. 4. Outage probabilities of SCUs versus number of base antennas $(M)$ for different transmit powers $\left(P_{S}\right)$.

\section{B. Outage probability of $S C U$}

In Fig. 5, the results of Monte Carlo simulation and analytical expressions for the outage probability of SCUs with $\varepsilon_{1}=5$ bits per channel use (BPCU), $\varepsilon_{2}=5 \mathrm{BPCU}$ and $P_{1}=P_{2}^{\prime}=P_{2} /$ 2 are presented where we set the signal to noise ratio (SNR) as $S N R=\frac{P_{1}}{N_{0}}=\frac{P_{2}^{\prime}}{N_{0}}=\frac{P_{2}}{2 N_{0}}$. The outage probabilities of near and far SCUs evaluated in Section IV for different SNRs show that analytical expressions match the simulation results well and the outage probabilities of near and far SCUs decrease as the SNR increases. Moreover, it is possible to observe that the outage probability of far user is lower than that of the near user, which shows the impact of proposed cooperative communication on far user.

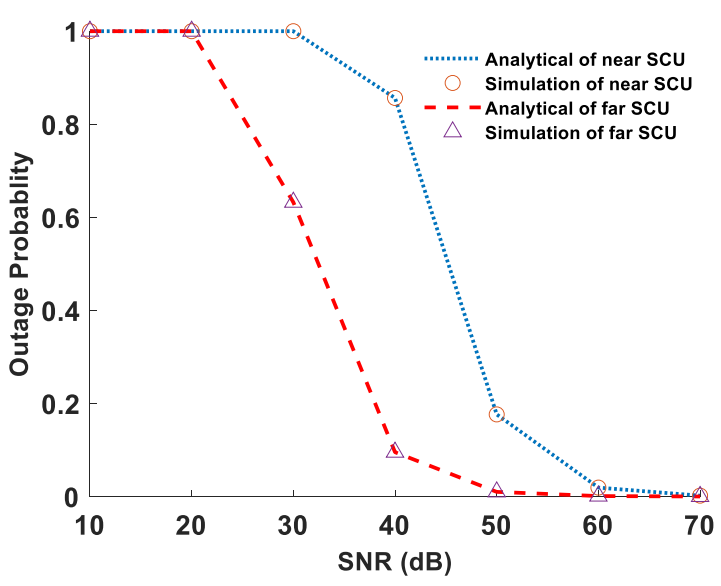

Fig. 5 Outage probabilities of SCU versus SNR for near and far SCUs.

In Fig. 6, the outage probabilities of near and far SCUs versus target data rate for different SNRs are shown. For the outage probability of near user, $\varepsilon_{2}$ is set to $0.5 \mathrm{BPCU}$ and $P_{1}=P_{2}^{\prime}=$ $P_{2} / 2$. As shown in Fig. 6(a), the outage probability of near user decreases by increasing SNR. Moreover, the lower target data rate, the lower is the outage probability as expected. In Fig. 6(b) instead, $\varepsilon_{1}$ is set to $0.75 \mathrm{BPCU}$ and $P_{1}=P_{2}^{\prime}=P_{2} / 10$. Also, far user acts in the same way by increasing target data rate and SNR. Analytical and simulation results match very well in all figures.

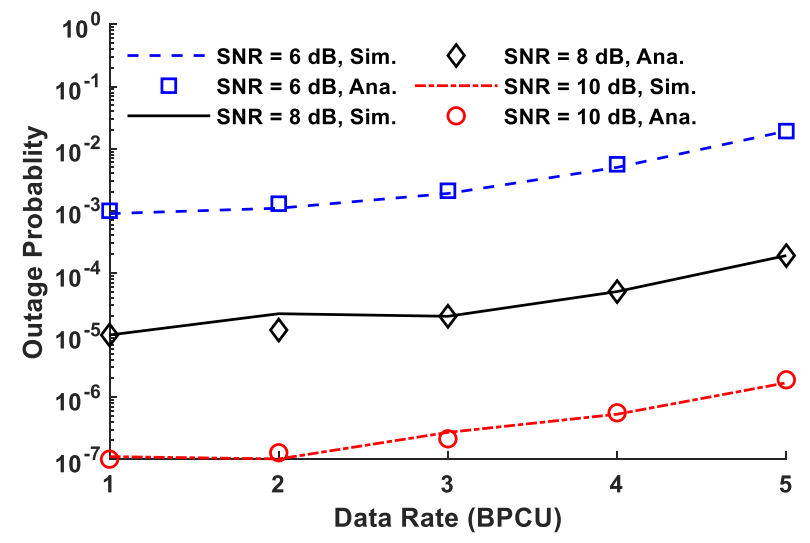

(a) near SCU

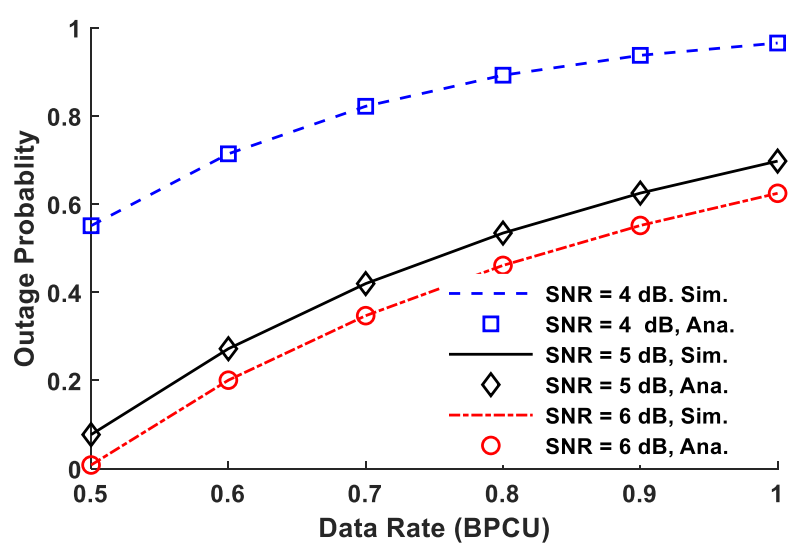

(b) far SCU

Fig. 6. Outage probability of near SCU versus data rate $\left(\varepsilon_{1}\right)$ and far SCU vs. data rate $\left(\varepsilon_{2}\right)$ for different transmit SNRs. 


\section{Performance evaluation of different grouping schemes}

In Fig. 7 and Fig. 8, the performance of cooperative mmWave-NOMA network with WPT for different grouping schemes is shown. We compare the proposed user grouping schemes with three reference user grouping schemes: $i$ ) random near random far (RNRF), in which both near and far users are randomly chosen from $\left\{A_{i}\right\}$ and $\left\{B_{j}\right\}$, respectively; ii) nearest near nearest far (NNNF), in which the nearest near user and the nearest far user are chosen from $\left\{A_{i}\right\}$ and $\left\{B_{j}\right\}$, respectively; and iii) nearest near furthest far (NNFF), in which the nearest near user and the farthest far user are chosen from $\left\{A_{i}\right\}$ and $\left\{B_{j}\right\}$, respectively [25]. In the RNRF, NNFF, and NNNF grouping methods, BA is employed for power allocation. As shown, the best grouping schemes in terms of SE for high SNR values are WFOF and WFSN, while for lower values their performance are near to the NNFF. Since, in the NNFF, the nearest near user is selected, and this user has the highest SE, with a very high probability this user is the one with the highest EE. Thus, it is the one selected in the proposed schemes. Moreover, among the compared schemes, the best grouping scheme for low SNRs is NNFF, since the nearest user from the near users group, with the farthest user from far users, result in high channel gain difference and achieving the best performance. For high SNRs, the performance of NNFF is very close to the NNNF where the nearest near user groups with the nearest far user. The reason is that in high SNRs, the effect of near user on the whole EE of system is more significant, and in both grouping methods the same near user is selected. The same result holds for the cumulative distribution function (CDF) of EE for different grouping schemes, as shown in Fig. 8. Finally, the proposed schemes are by far the best grouping schemes in terms of EE. Also, it has been shown that the sub-optimal user grouping (WFSN) achieved near optimal results with lower complexity.

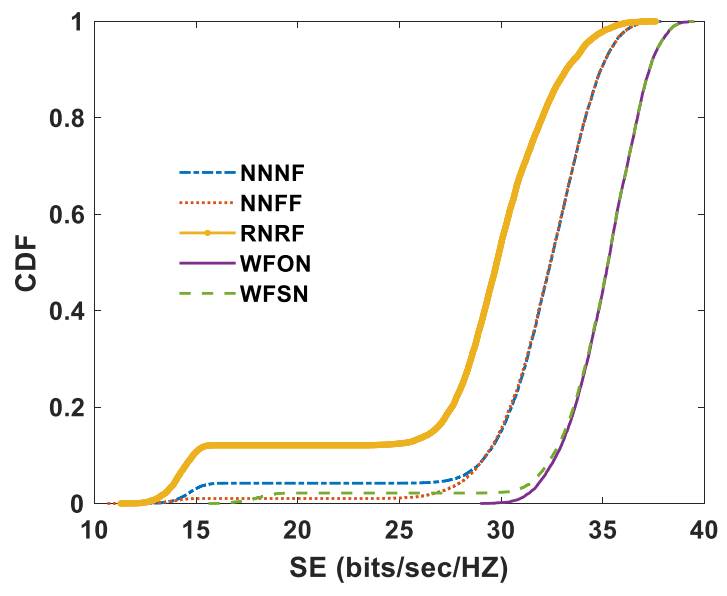

Fig. 7. CDF of SE for different grouping schemes.

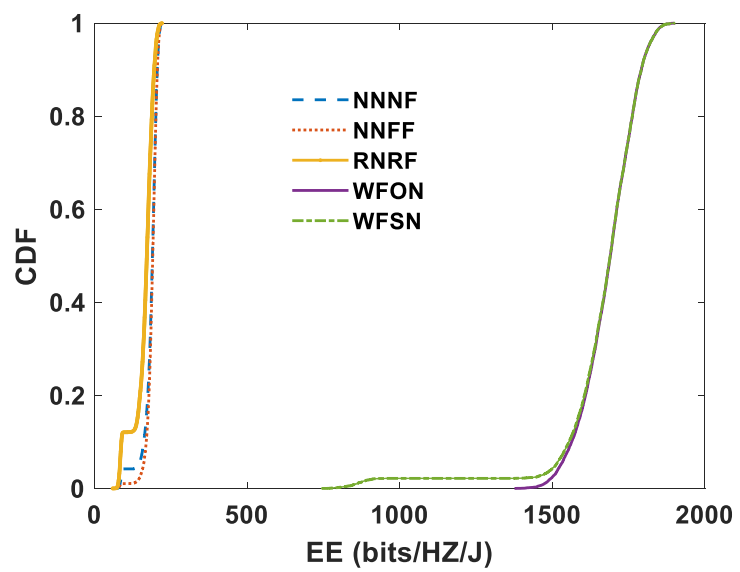

Fig. 8. CDF of EE for different grouping schemes.

\section{Performance comparison}

In order to illustrate the efficiency of the proposed system, we compare the performance in terms of SE and EE with other benchmarks solutions:

- WPT without cooperation (WWC): The time switching method is used to harvest energy. In this approach, in the first time slot the energy is harvested by the users from the BS and in the second time slot both users send their signals by conventional NOMA uplink method [47].

- Without WPT: In this approach only primary powers of near and far users are utilized for sending the superimpose messages of $U E_{1}$ and $U E_{2}$, and both direct and cooperative phases are employed.

- $\quad F-W P T$ : In this method, only far users harvest energy from BS and near users utilize primary powers for sending the superimpose message of $U E_{1}$. Both direct and cooperative phases are employed in this approach.

- $\quad N-W P T$ : In this scheme, energy harvesting mechanism is considered only for near users and both cooperative and direct phases are used for data transmission.

- Asynchronous transmission (AT): In this approach the users start harvesting energy at the same time, while information signals to the SBS is supposed to be transmitted at different time instants. The advantage of this approach is that the user with the best channel condition, which needs lower power level to transmit data, will be active first to send data to the SBS. Conversely, the far user with weak channel gain will harvest energy over a longer time interval and then use the rest of the duration to transfer information uplink [66].

In Fig. 9, the impact of WPT and cooperation on the CDFs of SE and EE is depicted. In all methods, NNFF is selected for user grouping and BA is used for power allocation of users. As shown in Fig. 9(a), the proposed approach with cooperation and WPT outperforms other methods. Without WPT denotes the condition that only the primary powers of users are utilized for 
data transmission, leading to degradation of the performance of cooperation because the near user acts as relay and less power is utilized for sending whole messages. WWC is less spectral efficient, because time switching method is used for WPT where the data and energy are transmitted orthogonally. In addition, in $W W C$, the signal is transmitted without cooperation which degrades the performance of system. WWC has almost similar performance than $A T$ since in both there is not any cooperation. However, for lower SE values, $W W C$ outperform $A T$, since in $A T$ a higher amount of time is consumed for the energy harvesting of the far user than $W W C$, and less time remains for data transmission, leading to a lower SE. For higher SE values, $A T$ outperforms $W W C$, since the $\mathrm{SE}$ of near user is more sensible and in $A T$ more time is consumed for data transmission of near user than the $W W C$, leading to higher SE. As depicted in Fig. 9(b), the CDF of EE of the proposed method outperforms WWC, without WPT and AT methods remarkably, confirming that deploying a cooperative network with WPT is efficient in mmWave-NOMA systems. Also, to confirm the importance of WPT on near and far users, Fig. 10 compares the CDFs of EEs of the proposed $F$-WPT and N-WPT methods. We observe that WPT on near user is more efficient than far user. This is due to the fact that near user plays the relay role and requires more power in comparison with the far user.

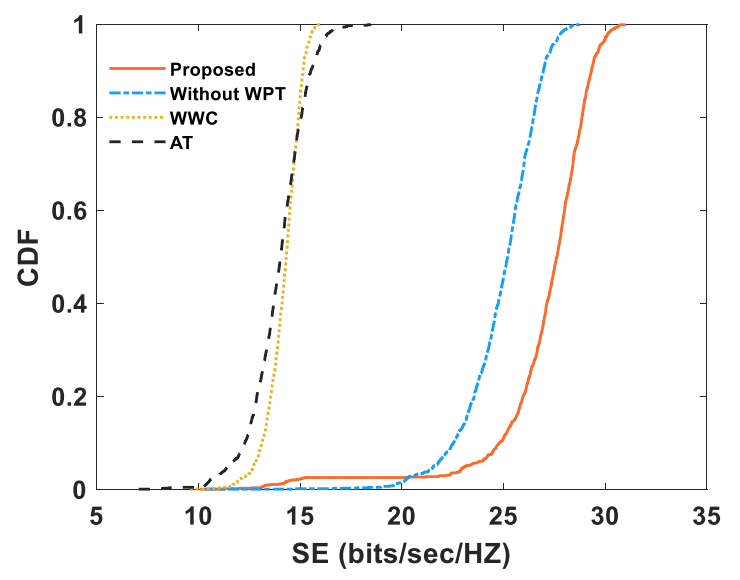

(a)

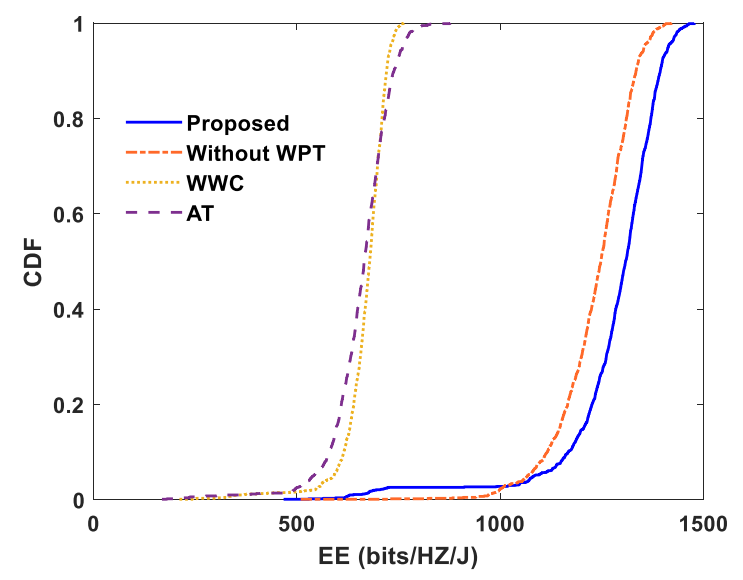

(b)

Fig. 9. CDF of (a) SE and (b) EE for different methods.

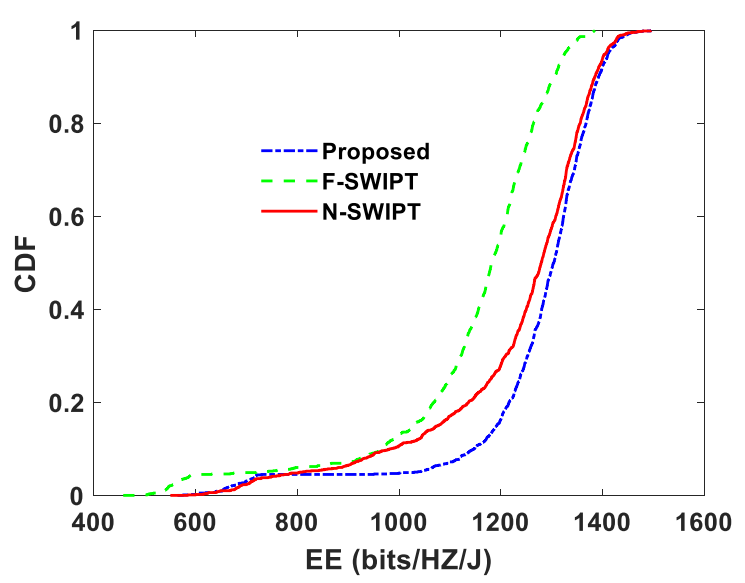

Fig. 10. CDF of EE for different methods.

In Fig. 11, the tradeoff between the EE and target data rates of $U E_{1}$ and $U E_{2}$ is depicted. As shown, the EE in the proposed and without WPT methods remain fixed until a certain target data rate and then drops. However, in the proposed method the EE drops at a higher data rate than the without WPT method. This is due to the fact that by increasing the target data rate of $U E_{1}$, more power is required for transmitting the message of $U E_{1}$, hence, the remaining power for cooperative transmission of $U E_{2}$ decreases. This drop occurs earlier for without WPT method because of the lower power budget. Moreover, the reduction of EE occurs at a lower data rate in $W W C$ and $A T$ methods, since in these methods there is no cooperation between the users, and increasing the power of one user has no impact on the others; thus, the imbalance between the target data rate and power consumption leads to the rapid reduction in the EE of system.

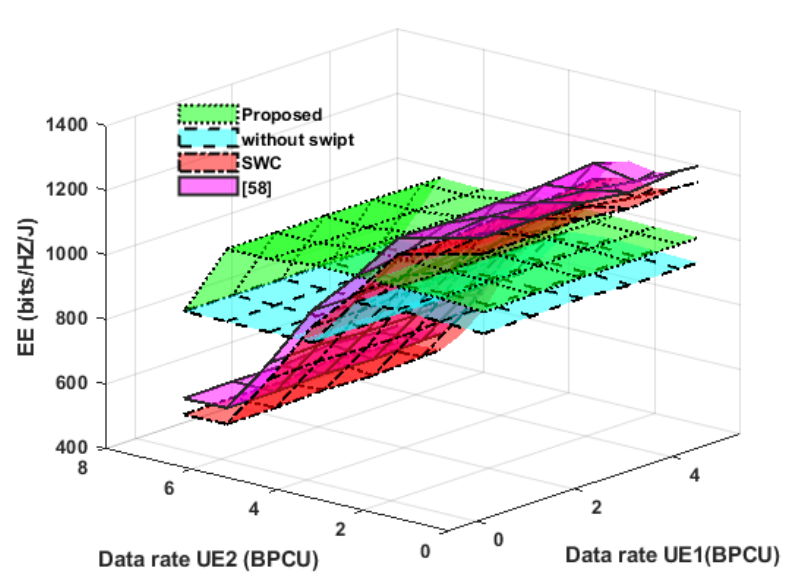

Fig. 11. EE of different systems versus $R_{U E_{1}}^{\min }$ and $R_{U E_{2}}^{\min }$.

\section{E. Performance evaluation of $B A$}

We demonstrate the effectiveness of BA in finding the optimum value of power in EE maximization problem, where 
users are grouped based on the NNFF scheme. In addition, the population size is a critical parameter in the BA. The lower population size may cause lack of diversity, and a higher population size may lead to slow convergence [67]. Then, we present the BA performance with different values of $b$ and compare the proposed BA solution with two other benchmarks as below:

- Exhaustive search: the optimum value of EE maximization problem is found by searching over all possible values of powers.

- Equal power: power allocation is performed equally as $P_{1}=P_{2}^{\prime}=P_{2}$.

As shown in Fig. 12, the proposed BA-based approach with $b=40$ and exhaustive search methods are close allowing BA to reach the optimal value, and both outperform equal power allocation remarkably. Furthermore, in Fig. 13, the convergence speed of the proposed EE optimization problem with BA is depicted, where the normalized $\mathrm{EE}$ is achieved by min-max normalization. It is shown that BA converges after 25 iterations. More analysis for convergence of BA was done in [68], where the authors proposed a Markov model for the algorithm and proved that the state sequence of the bat population forms a finite homogeneous Markov chain, satisfying the global convergence criteria.

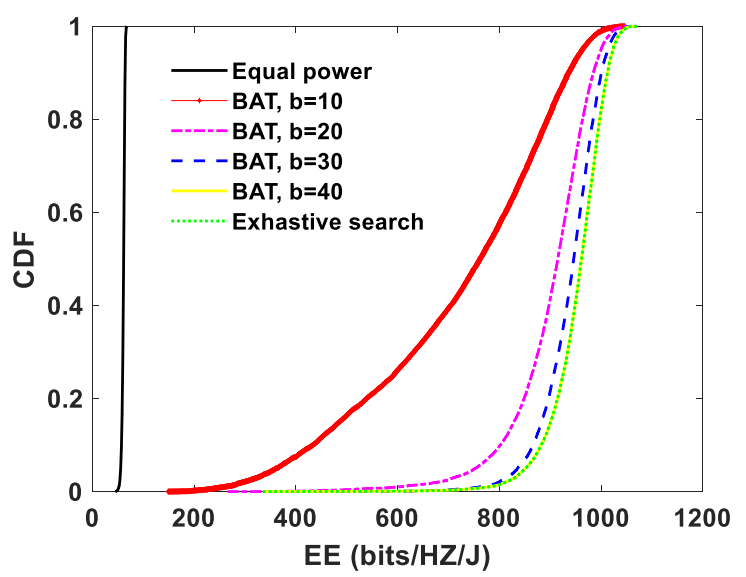

Fig. 12. CDF of EE for different power allocation methods.

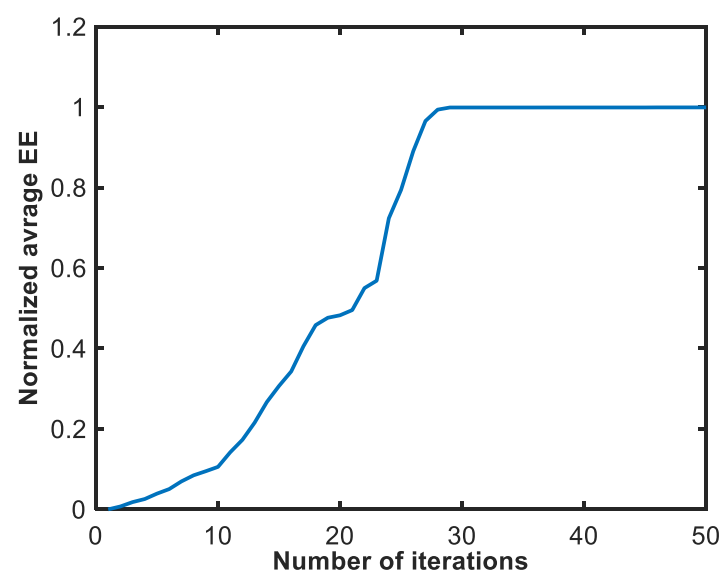

Fig. 13. Convergence speed of proposed EE optimization problem.

\section{F. Computational complexity of proposed power allocation} and user grouping methods

The computational complexity of the proposed power allocation method is obtained by considering different steps of $\mathrm{BA}$ as

1. In the initialization of different parameters of BA, the time complexity is of the order $O(2 b D+5 b)$, where $D$ denotes the number of optimization parameters which is three and $b$ shows the population size.

2. Evaluation of fitness values has the complexity of $O(b D)$.

3. Updating different parameters has the time complexity of $O(G b)$, where $G$ denotes the generation size.

By neglecting the complexity of other simple steps, the approximated computational complexity of the proposed algorithm is of $O(G b)$. It has to be noticed that the WFON has a complexity of $O(K L)$. Thus, the overall complexity of user grouping and power allocation is $O(G b \times K L)$. Moreover, for further reducing the computational complexity, WFSN is proposed where the user grouping is done based on the fixed power allocation. Hence, by neglecting the computational complexity of user grouping, the overall complexity is of $O(G b)$. This complexity is lower than some of the well-known algorithms in the literature such as the grid search algorithm [69], where user grouping and power allocation has a complexity of $O\left((K+L)^{(K+L)} \times N_{P}{ }^{(K+L)}\right)$, and $N_{P}$ is the number of possible transmit power values of users. while as shown before, it has approximately the same performance as the proposed method.

\section{CONCLUSIONS}

In this paper, we introduced a new cooperative NOMA with WPT protocol for uplink in mmWave network in order to maximize $\mathrm{SE}$ and $\mathrm{EE}$ of system. The formulated $\mathrm{EE}$ optimization problem was solved by decoupling the original problem in two sub-problems, where user grouping and power allocation problems were optimized independently. An iterative algorithm is proposed for user grouping. Transmit powers are allocated by using the BA and its efficiency was investigated through simulation results. In addition, closed form expressions for energy outage probability, outage probabilities and diversity order of users were derived which validate simulation results. We compared the proposed scheme with time switching-based method as $W W C$, without $W P T$ and $A T$ scenarios. We obtained the impact of user grouping on the SE and EE of the proposed system. The results demonstrate that EE performance achieved with the proposed user grouping scheme is better than the other methods. Moreover, it was shown that the energy harvesting at user side improves the $\mathrm{EE}$ of network. In addition, the efficiency of WPT on the near and far users were explored, and it was shown that the WPT on near user, that plays the relay role, is more efficient. Therefore, it is worthy to consider the joint optimization of user grouping and energy harvesting for WPT-based NOMA networks as the future work. 


\section{REFERENCES}

1. Lahsen-Cherif, I., Spectral and Energy Efficiency in $5 G$ Wireless Networks. 2016, Paris Saclay.

2. Qian, L.P., et al., Joint uplink base station association and power control for small-cell networks with non-orthogonal multiple access. IEEE Transactions on Wireless Communications, 2017. 16(9): p. 5567-5582.

3. Ghosh, A., et al., Heterogeneous cellular networks: From theory to practice. IEEE communications magazine, 2012. 50(6): p. 54-64.

4. Ismail, M., et al., Uplink decentralized joint bandwidth and power allocation for energy-efficient operation in a heterogeneous wireless medium. IEEE transactions on communications, 2015. 63(4): p. 1483-1495.

5. Niu, Y., et al., Energy-efficient scheduling for mmWave backhauling of small cells in heterogeneous cellular networks. IEEE Transactions on Vehicular Technology, 2016. 66(3): p. 2674-2687.

6. Liu, Y., et al., Cooperative non-orthogonal multiple access with simultaneous wireless information and power transfer. IEEE Journal on Selected Areas in Communications, 2016. 34(4): p. 938953.

7. Baghani, M., et al., Dynamic Non-Orthogonal Multiple Access and Orthogonal Multiple Access in 5G Wireless Networks. IEEE Transactions on Communications, 2019. 67(9): p. 6360-6373.

8. Sedaghat, M.A. and R.R. Müller, On user pairing in uplink NOMA. IEEE Transactions on Wireless Communications, 2018. 17(5): p. 3474-3486.

9. Higuchi, K. and A. Benjebbour, Non-orthogonal multiple access (NOMA) with successive interference cancellation for future radio access. IEICE Transactions on Communications, 2015. 98(3): p. 403-414.

10. Zhou, Y., V.W. Wong, and R. Schober, Dynamic decode-andforward based cooperative NOMA with spatially random users. IEEE Transactions on Wireless Communications, 2018. 17(5): p. 3340-3356.

11. Hoang, T.M., N.T. Tan, and S.-G. Choi. Analysis of partial relay selection in NOMA systems with RF energy harvesting. in 2018 2nd International Conference on Recent Advances in Signal Processing, Telecommunications \& Computing (SigTelCom). 2018. IEEE.

12. Hoang, T.M., et al., Optimizing duration of energy harvesting for downlink NOMA full-duplex over Nakagami-m fading channel. AEU-international Journal of Electronics and Communications, 2018. 95: p. 199-206.

13. Hoang, T.M., N.L. Van, and B.C. Nguyen, On the Performance of Energy Harvesting Non-Orthogonal Multiple Access Relaying System with Imperfect Channel State Information over Rayleigh Fading Channels. Sensors, 2019. 19(15): p. 3327.

14. Tran, H.-V., G. Kaddoum, and K.T. Truong, Resource allocation in SWIPT networks under a nonlinear energy harvesting model: Power efficiency, user fairness, and channel nonreciprocity. IEEE Transactions on Vehicular Technology, 2018. 67(9): p. 8466-8480.

15. Tran, H.-V. and G. Kaddoum, Robust design of AC computingenabled receiver architecture for SWIPT networks. IEEE Wireless Communications Letters, 2019. 8(3): p. 801-804.

16. Tran, T.X., et al., Nonlinear energy harvesting for millimeter wave networks with large-scale antennas. IEEE Transactions on Vehicular Technology, 2018. 67(10): p. 9488-9498.

17. Kamga, G.N. and S. Aïssa, Wireless power transfer in mmWave massive MIMO systems with/without rain attenuation. IEEE Transactions on Communications, 2018. 67(1): p. 176-189.

18. Khan, T.A., A. Alkhateeb, and R.W. Heath, Millimeter wave energy harvesting. IEEE Transactions on Wireless Communications, 2016. 15(9): p. 6048-6062.

19. Wang, L., et al., Wireless powered dense cellular networks: How many small cells do we need? IEEE Journal on Selected Areas in Communications, 2017. 35(9): p. 2010-2024.

20. Cui, J., et al., Optimal user scheduling and power allocation for millimeter wave NOMA systems. IEEE Transactions on Wireless Communications, 2017. 17(3): p. 1502-1517.

21. Wang, B., et al., Spectrum and energy-efficient beamspace MIMONOMA for millimeter-wave communications using lens antenna array. IEEE Journal on Selected Areas in Communications, 2017. 35(10): p. 2370-2382.
22. Ding, Z., P. Fan, and H.V. Poor, Random beamforming in millimeter-wave NOMA networks. IEEE access, 2017. 5: p. 76677681.

23. Dai, L., et al., Hybrid precoding-based millimeter-wave massive MIMO-NOMA with simultaneous wireless information and power transfer. IEEE Journal on Selected Areas in Communications, 2018. 37(1): p. 131-141.

24. Xiao, Z., et al., Joint power allocation and beamforming for nonorthogonal multiple access (NOMA) in $5 G$ millimeter wave communications. IEEE Transactions on Wireless Communications, 2018. 17(5): p. 2961-2974.

25. Lv, T., et al., Millimeter-wave NOMA transmission in cellular M2M communications for Internet of Things. IEEE Internet of Things Journal, 2018. 5(3): p. 1989-2000.

26. Marcano, A.S. and H.L. Christiansen, Impact of NOMA on network capacity dimensioning for $5 G$ HetNets. Ieee Access, 2018. 6: p. 13587-13603.

27. Zhu, L., et al., Joint power control and beamforming for uplink nonorthogonal multiple access in $5 G$ millimeter-wave communications. IEEE Transactions on Wireless Communications, 2018. 17(9): p. 6177-6189.

28. Hao, W., et al., Energy-efficient power allocation in millimeter wave massive MIMO with non-orthogonal multiple access. IEEE Wireless Communications Letters, 2017. 6(6): p. 782-785.

29. Han, T., et al., On downlink NOMA in heterogeneous networks with non-uniform small cell deployment. IEEE Access, 2018. 6: p. 31099-31109.

30. Zeng, M., et al., Energy-efficient power allocation in uplink mmWave massive MIMO with NOMA. IEEE Transactions on Vehicular Technology, 2019. 68(3): p. 3000-3004.

31. Zhu, L., et al., Millimeter-Wave NOMA with User Grouping, Power Allocation and Hybrid Beamforming. IEEE Transactions on Wireless Communications, 2019. 18(11): p. 5065-5079.

32. Yi, W., et al., Clustered millimeter-wave networks with nonorthogonal multiple access. IEEE Transactions on Communications, 2019. 67(6): p. 4350-4364.

33. Zaidi, S.K., S.F. Hasan, and X. Gui, Evaluating the ergodic rate in SWIPT-aided hybrid NOMA. IEEE Communications Letters, 2018. 22(9): p. 1870-1873.

34. Do, D.-T. and C.-B. Le, Application of NOMA in wireless system with wireless power transfer scheme: Outage and ergodic capacity performance analysis. Sensors, 2018. 18(10): p. 3501.

35. Pei, L., et al., Energy-efficient D2D communications underlaying NOMA-based networks with energy harvesting. IEEE Communications Letters, 2018. 22(5): p. 914-917.

36. Hedayati, M. and I.-M. Kim, On the performance of NOMA in the two-user SWIPT system. IEEE Transactions on Vehicular Technology, 2018. 67(11): p. 11258-11263.

37. Liu, Y., et al. Cooperative non-orthogonal multiple access in $5 G$ systems with SWIPT. in 2015 23rd European signal processing conference (EUSIPCO). 2015. IEEE.

38. Xu, Y., et al., Joint beamforming and power-splitting control in downlink cooperative SWIPT NOMA systems. IEEE Transactions on Signal Processing, 2017. 65(18): p. 4874-4886.

39. Su, B., Q. Ni, and W. Yu, Robust transmit beamforming for SWIPTenabled cooperative NOMA with channel uncertainties. IEEE Transactions on Communications, 2019. 67(6): p. 4381-4392.

40. Luo, J., et al., A deep learning-based approach to power minimization in multi-carrier NOMA with SWIPT. IEEE Access, 2019. 7: p. 17450-17460.

41. Yuan, Y., et al., Energy efficiency optimization in full-duplex useraided cooperative SWIPT NOMA systems. IEEE Transactions on Communications, 2019. 67(8): p. 5753-5767.

42. Nguyen, T.N., P.T. Tran, and M. Vozňák, Power splitting - based energy - harvesting protocol for wireless - powered communication networks with a bidirectional relay. International Journal of Communication Systems, 2018. 31(13): p. e3721.

43. Cheng, X. and Y. Liu. Uplink simultaneous wireless information and power transfer with non-orthogonal multiple access. in 2017 9 th International Conference on Wireless Communications and Signal Processing (WCSP). 2017. IEEE. 
44. Nasir, A.A., et al., Relaying protocols for wireless energy harvesting and information processing. IEEE Transactions on Wireless Communications, 2013. 12(7): p. 3622-3636.

45. Tang, J., et al., Energy efficiency optimization for NOMA with SWIPT. IEEE Journal of Selected Topics in Signal Processing, 2019. 13(3): p. 452-466.

46. Kader, M.F., et al., Cooperative non - orthogonal multiple access with SWIPT over Nakagami - $m$ fading channels. Transactions on Emerging Telecommunications Technologies, 2019. 30(5): p. e3571.

47. Zaidi, S.K., S.F. Hasan, and X. Gui. SWIPT-aided uplink in hybrid non-orthogonal multiple access. in 2018 IEEE Wireless Communications and Networking Conference (WCNC). 2018. IEEE.

48. Krikidis, I., et al., Simultaneous wireless information and power transfer in modern communication systems. IEEE Communications Magazine, 2014. 52(11): p. 104-110.

49. Chen, Y., Energy Harvesting Communications: Principles and Theories. 2019: John Wiley \& Sons.

50. Zeng, Y., H. Chen, and R. Zhang, Bidirectional wireless information and power transfer with a helping relay. IEEE Communications Letters, 2016. 20(5): p. 862-865.

51. Aldababsa, M. and O. Kucur. Outage performance of NOMA with TAS/MRC in dual hop AF relaying networks. in 2017 Advances in Wireless and Optical Communications (RTUWO). 2017. IEEE.

52. Ali, M.S., H. Tabassum, and E. Hossain, Dynamic user clustering and power allocation for uplink and downlink non-orthogonal multiple access (NOMA) systems. IEEE access, 2016. 4: p. 63256343.

53. Lee, S. and J.H. Lee. Joint User Scheduling and Power Allocation for Energy Efficient Millimeter Wave NOMA Systems With Random Beamforming. in 2018 IEEE 88th Vehicular Technology Conference (VTC-Fall). 2018. IEEE.

54. Khazali, A., et al., Energy-spectral efficient resource allocation and power control in heterogeneous networks with D2D communication. Wireless Networks, 2018: p. 1-15.

55. Duan, W., et al., Capacity enhanced cooperative D2D systems over rayleigh fading channels with NOMA. arXiv preprint arXiv:1810.06837, 2018.

56. Fayaz, M. and D. Kim, Energy consumption optimization and user comfort management in residential buildings using a bat algorithm and fuzzy logic. Energies, 2018. 11(1): p. 161.

57. Gandomi, A.H., et al., Bat algorithm for constrained optimization tasks. Neural Computing and Applications, 2013. 22(6): p. 12391255 .

58. Reddy, P.S.K., P. Kumar, and G. Vaibhav, Application of BAT algorithm for optimal power dispatch. Int J Innov Res Adv Eng, 2015. 2(2): p. 113-119.

59. Yang, X.-S., A new metaheuristic bat-inspired algorithm, in Nature inspired cooperative strategies for optimization (NICSO 2010). 2010, Springer. p. 65-74.

60. Psomas, C. and I. Krikidis, Energy beamforming in wireless powered mmwave sensor networks. IEEE Journal on Selected Areas in Communications, 2018. 37(2): p. 424-438.

61. Liu, Y., M. Derakhshani, and S. Lambotharan, Outage analysis and power allocation in uplink non-orthogonal multiple access systems. IEEE Communications Letters, 2017. 22(2): p. 336-339.

62. Tang, W., S. Kang, and B. Ren, Performance analysis of cooperative pattern division multiple access (Co-PDMA) in uplink network. IEEE Access, 2017. 5: p. 3860-3868.

63. Yang, Z., et al., A general power allocation scheme to guarantee quality of service in downlink and uplink NOMA systems. IEEE transactions on wireless communications, 2016. 15(11): p. 72447257.

64. Li, Y., et al., Performance analysis of cooperative NOMA with a shared AF relay. IET Communications, 2018. 12(19): p. 2438-2447.

65. Wang, X., et al., Exploiting full-duplex two-way relay cooperative non-orthogonal multiple access. IEEE Transactions on Communications, 2018. 67(4): p. 2716-2729.

66. Zewde, T.A. and M.C. Gursoy, NOMA-based energy-efficient wireless powered communications. IEEE Transactions on Green Communications and Networking, 2018. 2(3): p. 679-692.
67. Fister, I., U. Mlakar, and X.-S. Yang, Parameterless bat algorithm and its performance study, in Nature-inspired computation in engineering. 2016, Springer. p. 267-276.

68. Chen, S., et al., Global convergence analysis of the bat algorithm using a markovian framework and dynamical system theory. Expert Systems with Applications, 2018. 114: p. 173-182.

69. Zhang, J., et al., Optimal and Sub-Optimal Uplink NOMA: Joint User Grouping, Decoding Order, and Power Control. IEEE Wireless Communications Letters, 2019. 9(2): p. 254-257. 